\title{
Passive scalar decay in chaotic flows with boundaries
}

\author{
Fatma A. Zaggout and Andrew D. Gilbert \\ Mathematics Research Institute, \\ College of Engineering, Mathematics and Physical Sciences, \\ University of Exeter, U.K.
}

\begin{abstract}
This paper considers the long-time decay rate of a passive scalar in two-dimensional flow. The focus is on the effects of boundary conditions for kinematically prescribed velocity fields with random or periodic time dependence. Scalar evolution is followed numerically in a periodic geometry for families of flows that have either a slip or a no-slip boundary condition on a square or plane layer subdomain $\mathcal{D}$. The boundary conditions on the passive scalar are imposed on the boundary of $\mathcal{D}$ by restricting to a subclass invariant under certain symmetry transformations. The scalar field obeys constant (Dirichlet) or no-flux (Neumann) conditions exactly for a flow with the slip boundary condition and approximately in the no-slip case.

At late times the decay of a passive scalar is exponential in time with a decay rate $\gamma(\kappa)$, where $\kappa$ is the molecular diffusivity. Scaling laws of the form $\gamma(\kappa) \simeq$ $C \kappa^{\alpha}$ for small $\kappa$ are obtained numerically for a variety of boundary conditions on flow and scalar, and supporting theoretical arguments are presented. In particular when the scalar field satisfies a Neumann condition on all boundaries, $\alpha \simeq 0$ for a slip flow condition; for a no-slip condition we confirm results in the literature that $\alpha \simeq 1 / 2$ for a plane layer, but find $\alpha \simeq 2 / 3$ in a square subdomain $\mathcal{D}$ where the decay is controlled by stagnant flow in the corners. For cases where there is a Dirichlet boundary condition on one or more sides of the subdomain $\mathcal{D}$, the exponent measuring the decay of the scalar field is $\alpha \simeq 1 / 2$ for a slip flow condition and $\alpha \simeq 3 / 4$ for a no-slip condition. The scaling law exponents $\alpha$ for chaotic timeperiodic flows are compared with those for similarly constructed random flows.
\end{abstract}

Key words: chaotic advection, boundary conditions, passive scalar 


\section{Introduction}

The problem of how a passive scalar is mixed by a fluid flow remains important for theory and applications. Understanding mixing and enhanced diffusion processes in deterministic, random and turbulent flows is key in applications such as chemical engineering and combustion (e.g. Ottino, 1989), and areas including transport in atmospheres and oceans (e.g., Pierrehumbert \& Yang, 1993). In the limit of weak scalar diffusivity $\kappa$, the behaviour of the scalar probes the Lagrangian complexity of the flow, in particular the presence of intermingled islands and chaotic regions in the case of time-periodic, chaotic flow (Aref, 1984). It is important to note that this complexity can occur in the Lagrangian particle trajectories of flows that are regular from the Eulerian viewpoint, for example time-periodic flows in two dimensions or even steady flows in three. Mixing has been studied in many idealised models: in deterministic or random flows which exhibit global chaos, so that no integrable islands are present, the decay of a passive scalar is exponential, at a rate $\gamma(\kappa)$ that tends to a constant independent of the diffusivity as $\kappa \rightarrow 0$ (e.g., Toussaint et al., 2000). In some situations the decay rate may be considered as being determined by the spectrum of Liapunov exponents in the flow (Antonsen et al., 1996), but when the flow includes many scales or when the scalar field is allowed to adopt larger scales than the flow, the decay is instead controlled by passive scalar transport over these scales (Fereday \& Haynes, 2004; Schekochihin, Haynes \& Cowley, 2004). The competition between these two effects, stretching versus transport, has been understood in terms of the spectra of linear operators (Haynes \& Vanneste, 2005). Another viewpoint introduces the notion of a 'strange' eigenfunction (Pierrehumbert, 1994) in which as the diffusivity $\kappa$ tends to zero, a scalar field takes a fractal form, the same at larger scales, but acquiring structure on progressively finer scales in this limit. For a finite diffusivity, the long-time scalar field is a smoothed version of the strange eigenfunction defined for $\kappa=0$ with structure on all scales, an idea that can be made rigorous for idealised models based on baker's maps (e.g. Gilbert, 2006).

In the present paper our interest is where boundary conditions on the fluid flow may be important for the evolution and decay of passive scalars.. Here we have in mind flows which obey a realistic no-slip condition $\boldsymbol{u}=0$ on the boundary $\mathcal{C}$ of a fluid domain $\mathcal{D}$, or have the more idealised slip condition in which only the absence of normal flow is specified, $\boldsymbol{n} \cdot \boldsymbol{u}=0$. There has been much interest in this topic recently, in particular building on the work of Chertkov \& Lebedev (2003), Lebedev \& Turitsyn (2004) and Chernykh \& Lebedev (2008). These authors make the key observation that near to a no-slip boundary the effective time scale of mixing becomes very long. The resulting boundary layer can control the mixing of passive scalar by acting as a reservoir, slowly releasing scalar into the body of the flow, where mixing occurs more rapidly. If the flow is random, then near the boundary the effective correlation time of the flow becomes very short (as the turnover time increases). Approximation leads to a diffusion equation determining the boundary layer structure and giving predictions about decay rates and scalar field moments.

This has been further explored by Salman \& Haynes (2007) who use families of 'renewing' flows, in which the random flow is chosen to be independent and identically distributed on time intervals $[j \tau,(j+1) \tau]$. These authors identify several distinct regimes in the mixing and decay process, in agreement with Lebedev \& Turitsyn (2004). After rapid mixing in the bulk of the fluid, in an intermediate regime the slow release of tongues of relatively large scale scalar from 
the boundaries leads to a slow decay of concentration variance, discussed from the point of view of experiment and theory by Gouillart et al. $(2007,2008)$ and Gouillart, Thiffeault \& Dauchot (2010). The theory of Lebedev \& Turitsyn (2004) has been supported by numerical simulations of scalar transport in three-dimensional Couette flow by Boffetta, De Lillo \& Mazzino (2009) and extended to the case of turbulent flow, where the wall layer has a more complex structure (Garcia-Ybarra, 2009; Skvortsov \& Yee, 2011). This is for flows in closed systems, bounded regions of the plane or a plane layer with periodic boundary conditions. A further element of the study of Lebedev \& Turitsyn (2004) concerns the decay of scalar variance in the open system consisting of flow down a pipe and the influence of no-slip boundary conditions; this geometry allows experimental verification of theory, and is particularly important in mixing applications for microfluidics (Groisman \& Steinberg, 2001; Burghelea et al., 2004; Burghelea, Segre \& Steinberg, 2004; Simonnet \& Groisman, 2005; Jun \& Steinberg, 2010).

The focus of the above studies for closed systems is the case of the decay of passive scalar fluctuations for flows obeying a no-slip condition, where the scalar obeys a no-flux or Neumann condition, in a plane layer domain with periodic boundary conditions along the layer. In this case the long-time regime is exponential decay with a decay rate $\gamma(\kappa)$ proportional to $\kappa^{1 / 2}$. The aim of our paper is to look at other cases and to survey the different types of scaling that may occur. We consider flows in a plane layer domain and also in a square domain possessing corners: here the flow is even slower than that near a no-slip boundary condition, and we are interested in the effect this may have. We consider flows with both slip and no-slip conditions, and scalar fields with Neumann and Dirichlet conditions. It will sometimes be useful to think of the passive scalar as temperature for the sake of discussion, although we are not considering convection and our results apply to any passive scalar. In the first case of a Neumann boundary condition, the focus is on the mixing and homogenisation of temperature within the domain $\mathcal{D}$ and how fast fluctuations are destroyed. In the second case of a Dirichlet boundary condition, the aim is to understand how rapidly heat leaves the domain and so how fast the fluid cools; as heat must cross the bondaries, boundary layers are crucial in controlling the flux (cf. Balmforth \& Young, 2003). In each case our focus is on the long-time behaviour of the passive scalar, with decay rates scaling as $\gamma(\kappa) \simeq C \kappa^{\alpha}$ in the limit of small $\kappa$ for an exponent $\alpha$ depending on the boundary conditions. The Dirichlet case has been little studied despite its importance in linking questions of chaotic advection to heat transfer, and possible applications. However the recent paper El Omari \& Le Guer (2010) considers decay of temperature for a two-rod mixing device under various protocols for rotating the rods. The full Navier-Stokes equation for the fluid flow is solved, and it is found that the heat transfer is controlled by boundary layers on the no-slip boundaries.

We proceed in section 2 by defining families of flows with slip and no-slip boundary conditions on the boundary $\mathcal{C}$ of a square domain $\mathcal{D}$. In order to make use of an efficient numerical method we embed this domain in a region which is $2 \pi$ periodic in $x$ and $y$ (see figure 1 (a) below). By suitable combinations of Fourier modes we can easily write down flows that have zero normal flow $\boldsymbol{n} \cdot \boldsymbol{u}=0$ or obey no-slip $\boldsymbol{u}=0$ on the boundary $\mathcal{C}$ of $\mathcal{D}$. We then simulate passive scalars in these flows using a code which is completely spectral. We note that several other studies have used flows with $2 \pi$-periodicity, but with a focus on the presence of islands and other structures in the fluid flow, rather than on boundary conditions for the fluid flow and scalar (Cerbelli, Adrover \& Giona, 2003; Giona et al., 2004; Camassa, Martinsen-Burrell \& McLaughlin, 2007; 
Turner, Thuburn \& Gilbert, 2009). Crucial in our study is the use of symmetries: if the flow and initial condition are chosen suitably then evolution under the advection-diffusion equation preserves the Dirichlet or Neumann boundary conditions exactly for the case of slip flow, or approximately in the case of no-slip flow. In section 3 we simulate scalar advection and diffusion in the square domain $\mathcal{D}$ for random, renewing flows with a variety of boundary conditions. To make contact with previous work and to assess the role of domain shape, in section 4 we consider random flows in a plane layer geometry, making use of Bloch wavenumbers within our numerical code. The scaling exponents $\alpha$ that we obtain show values close to simple fractions, and in section 5 we link these exponents to theory of Lebedev \& Turitsyn (2004) and extensions. We also briefly present the results of some simulations of periodic flows, to investigate the validity of scaling behaviour $\gamma \simeq C \kappa^{\alpha}$ and compare exponents between random and deterministic cases. Finally, section 6 offers final discussion and suggestions for the extension of our study.

\section{Governing equations and symmetries}

We consider the advection of a passive scalar field $\sigma(\boldsymbol{r}, t)$ governed by the equation

$$
\frac{\partial \sigma}{\partial t}+\boldsymbol{u} \cdot \nabla \sigma=\kappa \nabla^{2} \sigma
$$

where $\boldsymbol{u}$ is a specified incompressible flow velocity, so that $\nabla \cdot \boldsymbol{u}=0$, and $\kappa>0$ is the diffusion coefficient. We take a non-dimensional formulation with $\boldsymbol{u}$ periodic in $(2 \pi)^{2}$ space $\mathbb{T}^{2}$, coordinates $\boldsymbol{r}=(x, y)$, and $\kappa \equiv \mathrm{Pe}^{-1}$ as the inverse Péclet or Schmidt number. The magnitude of scalar fluctuations in the periodic domain may be measured by the mean-square value,

$$
V(t)=(2 \pi)^{-2} \int_{-\pi}^{\pi} \int_{-\pi}^{\pi} \sigma^{2}(\boldsymbol{r}, t) d x d y
$$

(In fact this is the variance of the scalar in the full domain $\mathbb{T}^{2}$ as the mean is zero for the simulations below.) With $\kappa>0$, for a given flow the scalar has the long-time exponential decay rate $\gamma(\kappa)>0$ defined by

$$
\gamma=-\frac{1}{2} \lim _{t \rightarrow \infty} t^{-1} \log V(t)
$$

We consider flows $\boldsymbol{u}$ defined by a stream function $\psi$

$$
\boldsymbol{u}=\left(\partial_{y} \psi,-\partial_{x} \psi\right)
$$

with a Fourier decomposition

$$
\psi(\boldsymbol{r}, t)=\sum_{j, k} \hat{\psi}_{j, k}(t) e^{i j x+i k y}, \quad \hat{\psi}_{j, k}^{*} \equiv \hat{\psi}_{-j,-k}
$$

and likewise for the scalar field,

$$
\sigma(\boldsymbol{r}, t)=\sum_{m, n} \hat{\sigma}_{m, n}(t) e^{i m x+i n y}, \quad \hat{\sigma}_{m, n}^{*} \equiv \hat{\sigma}_{-m,-n}
$$



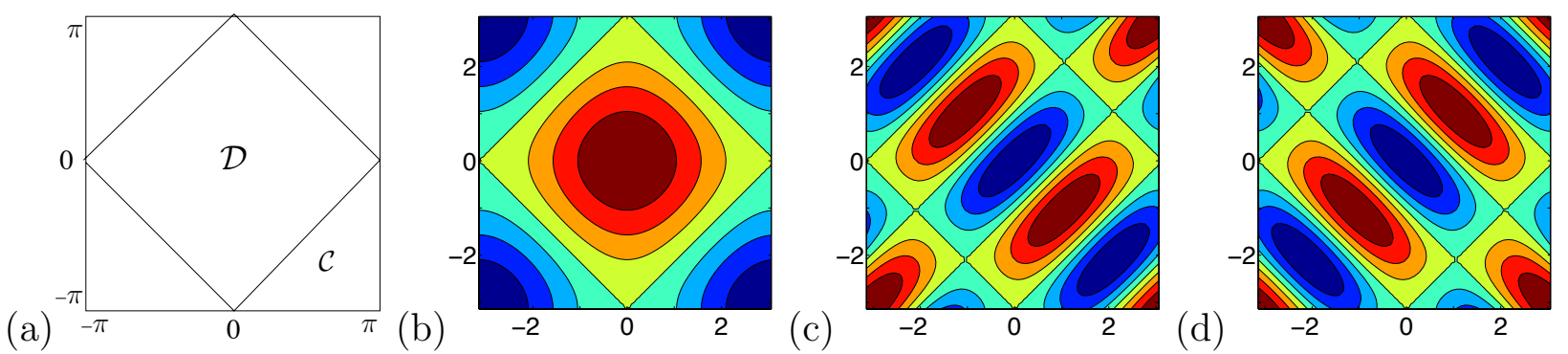

Fig. 1. Within $\mathbb{T}^{2}$ are depicted (a) the square $\mathcal{C}$ and the domain $\mathcal{D}$, together with the functions (b) $\phi_{1,1}$, (c) $\phi_{1,3}$ and (d) $\phi_{3,1}$.

in periodic space. The flows used are chosen to have relatively few non-zero Fourier modes $\hat{\psi}_{j, k}$. The numerical codes exploit this by keeping the scalar field in Fourier space (except for plotting), retaining up to $256^{2}$ modes for the larger runs, and evaluating the convolution term $\boldsymbol{u} \cdot \nabla \sigma$ explicitly. Time-stepping is done by the Adams-Bashforth scheme for the $\boldsymbol{u} \cdot \nabla \sigma$ term with the diffusion term integrated exactly.

Our aim is to impose no-slip or slip boundary conditions for the velocity field on the boundary $\mathcal{C}$ of a domain $\mathcal{D}$ in the fluid. We do this within the $2 \pi$ periodic space $\mathbb{T}^{2}$ by building fields that satisfy certain symmetry properties. Consider the square $\mathcal{C}$ with vertices at $(\pi, 0),(0, \pi),(-\pi, 0)$ and $(0,-\pi)$ depicted in figure $1(\mathrm{a})$ and call its interior the domain $\mathcal{D}$. We define functions

$$
\phi_{m, n}=2 \sin \left[\frac{m}{2}(x+y+\pi)\right] \sin \left[\frac{n}{2}(y-x+\pi)\right] .
$$

These are zero on the straight lines constituting $\mathcal{C}$, namely

$$
x+y= \pm \pi, \quad y-x= \pm \pi,
$$

and provided $m+n$ is even the fields $\phi_{m, n}$ are $2 \pi$-periodic. We thus use the family

$$
\Phi_{\text {even }}=\left\{\phi_{m, n}: m+n \text { is even }\right\}
$$

and in particular the members

$$
\begin{aligned}
& \phi_{1,1}=\cos x+\cos y, \\
& \phi_{1,3}=-\cos (2 x-y)-\cos (2 y-x), \\
& \phi_{3,1}=-\cos (2 x+y)-\cos (x+2 y),
\end{aligned}
$$

depicted in figure $1(\mathrm{~b}, \mathrm{c}, \mathrm{d})$ to compose periodic fields and flows satisfying boundary conditions on the boundary $\mathcal{C}$ of $\mathcal{D}$.

In order to do this, we need to consider possible symmetries of the flows and scalar fields. The square $\mathcal{C}$ embedded in periodic space $\mathbb{T}^{2}$, as depicted in figure $1(\mathrm{a})$, is invariant under a finite group $G$ of transformations: we stress that coordinates are taken modulo $2 \pi$ in both $x$ and $y$ directions. The group $G$ may be generated by a rotation $R$ of $\pi / 2$ about the origin, a reflection $S$ in the $x$-axis, and a diagonal translation $T$,

$$
R(x, y)=(-y, x), \quad S(x, y)=(x,-y), \quad T(x, y)=(x+\pi, y+\pi) .
$$


The first two of these generate the dihedral group $D_{8}$, acting on the square $\mathcal{C}$ in the standard fashion. As the translation $T$ commutes with $R$ and $S$ (modulo $2 \pi$ ), the full group is $G=$ $D_{8} \times \mathbb{Z}_{2}$.

Under a transformation $g \in G$, a $2 \pi$-periodic function $\phi(x, y)$ transforms according to

$$
(g \phi)(x, y)=\phi\left[g^{-1}(x, y)\right]
$$

giving, explicitly, for $\phi_{m, n} \in \Phi_{\text {even }}$,

$$
R \phi_{m, n}=(-1)^{n+1} \phi_{n, m}, \quad S \phi_{m, n}=\phi_{n, m}, \quad T \phi_{m, n}=(-1)^{m} \phi_{m, n} .
$$

Symmetries we will find particularly useful are reflections in the lines forming $\mathcal{C}$. Taking points modulo $2 \pi$ there are just two of these, namely reflection $S_{1}$ in (either of) the lines $x+y= \pm \pi$ and reflection $S_{2}$ in $y-x= \pm \pi$ given by

$$
\begin{aligned}
& S_{1}=T R^{3} S, \quad S_{1}(x, y)=(\pi-y, \pi-x), \\
& S_{2}=T R S, \quad S_{2}(x, y)=(y-\pi, x+\pi) .
\end{aligned}
$$

One may check that

$$
S_{1} \phi_{m, n}=S_{2} \phi_{m, n}=-\phi_{m, n} .
$$

We have thus specified 16 transformations, members of the group $G$, under which the basic geometry depicted in figure 1(a) is invariant. We will use these transformations to specify flows and scalar fields with slip/no-slip or Dirichlet/Neumann properties on the boundary $\mathcal{C}$, by requiring that these flows or scalar fields are invariant or change sign under certain transformations in $G$. These flows and fields will not transform simply under all elements of $G$, but only under a subgroup for each case; we will explain this further below.

\section{Simulations of flows in the square domain $\mathcal{D}$}

We use the above family $\Phi_{\text {even }}$ to define flows and simulate the evolution of passive scalar fields from an initial condition $\sigma_{0}(\boldsymbol{r})$ according to

$$
\frac{\partial \sigma}{\partial t}=J(\psi, \sigma)+\kappa \nabla^{2} \sigma
$$

where $J$ is the usual Jacobian. If under a transformation $g \in G$ we have that

$$
J \rightarrow s_{J} J, \quad \psi \rightarrow s_{\psi} \psi, \quad \sigma_{0} \rightarrow s_{\sigma} \sigma_{0}
$$

where each sign $s= \pm 1$, then the evolution equation preserves the property $\sigma \rightarrow s_{\sigma} \sigma$ under $g$ at future times, provided only that

$$
s_{J} s_{\psi}=1 .
$$

Here $s_{J}=-1$ if $g$ is a reflection and 1 if $g$ preserves orientation. 
(a)

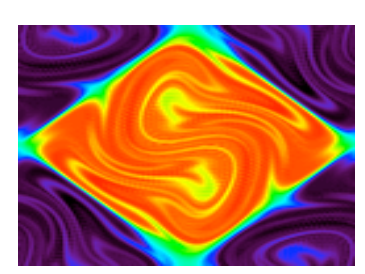

(b)

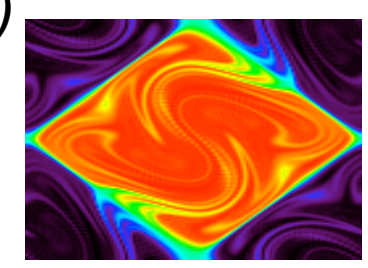

(c)

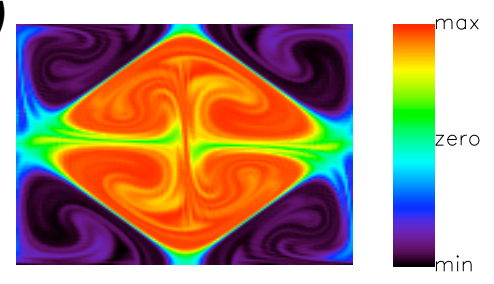

Fig. 2. Scalar field for $\left(\psi_{\text {slip }}, \sigma_{\mathrm{D}}\right)$ with $\kappa=0.001$ at (a) $t=20$, (b) 40 and (c) 60 . In the colour scale values go from black (minimum) to red (maximum) with zero green.

\subsection{Numerical simulations for the flow $\psi_{\text {slip }}$}

First we define a flow which has a free-slip boundary condition, given by the stream function

$$
\psi_{\text {slip }}=\phi_{1,3}(x, y) \sin [\omega t+\chi(t)]+\phi_{3,1}(x, y) \cos [\omega t+\chi(t)] .
$$

Here $\chi(t)$ is a phase that can be a constant, say zero, giving a time-periodic flow, or depend randomly on time as we detail later. This flow has stream function $\psi_{\text {slip }}=0$, constant on $\mathcal{C}$, so there is no normal flow on this boundary, $\boldsymbol{u} \cdot \boldsymbol{n}=0$. There is generally a slip velocity $\boldsymbol{u} \neq 0$, except at the corners of $\mathcal{C}$, where necessarily $\boldsymbol{u}=0$. We also define initial conditions $\sigma(\boldsymbol{r}, 0)=\sigma_{0}(\boldsymbol{r})$ for simulations of the scalar field, and focus on two,

$$
\sigma_{\mathrm{D}}=\phi_{1,1}, \quad \sigma_{\mathrm{N}}=\phi_{1,1}^{2}-1 .
$$

Both of these fields have zero mean in the full $2 \pi$ periodic domain. The former, shown in figure $1(\mathrm{~b})$, has non-zero average in the square subdomain $\mathcal{D}$ whereas for the latter the average is zero. Under the reflections about the boundaries of $\mathcal{C}$, we have

$$
S_{1}, S_{2}: \quad J \rightarrow-J, \quad \psi \rightarrow-\psi, \quad \sigma \rightarrow \mp \sigma,
$$

where the upper sign is for $\sigma_{\mathrm{D}}$ and the lower for $\sigma_{\mathrm{N}}$. Thus equation (3.3) is satisfied and these symmetry properties are preserved under subsequent evolution. As $\sigma_{\mathrm{D}}$ is odd under the reflections (upper sign), its values are zero on the boundary $\mathcal{C}$ and so it obeys a Dirichlet boundary condition $\sigma_{\mathrm{D}}=0$. Considering the passive scalar as temperature, the decay rates we will determine give a measure of how efficient a flow with slip boundary condition is at cooling a fluid through transfer of heat through the boundary. On the other hand $\sigma_{\mathrm{N}}$ is even under reflections and so must have zero perpendicular gradient $\boldsymbol{n} \cdot \nabla \sigma_{\mathrm{N}}=0$ on $\mathcal{C}$ : this is a Neumann boundary condition and (given that $\sigma_{\mathrm{N}}$ has zero mean in $\mathcal{D}$ ) the decay of its mean-square value $V(t)$ gives a measure of how efficient the flow is at destroying temperature fluctuations in the fluid in the presence of insulating boundaries.

We have investigated these mixing processes by means of numerical simulations and we begin by considering the case of $\psi_{\text {slip }}$ with Dirichlet boundary condition. We use (3.4) to create a random, 'renewing' flow by selecting $\chi(t)$ to be a piecewise constant random function with values on time-intervals $j \tau \leq t<(j+1) \tau$ distributed uniformly and independently between 0 and $2 \pi$ (Salman \& Haynes, 2007). We have taken $\tau=0.64$ and $\omega=1$ in this case and in all subsequent random flows. Figure 2 shows snapshots of the evolution of a scalar field in a simulation of one realisation of this random flow, using $\sigma_{\mathrm{D}}$ in (3.5) as initial condition to 


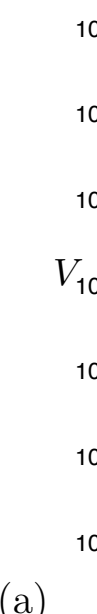

(a)

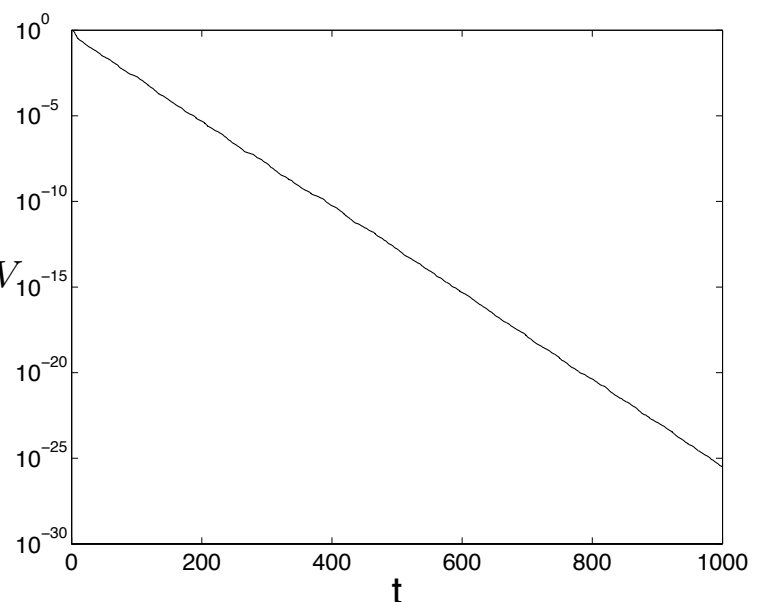

$\mathrm{t}$

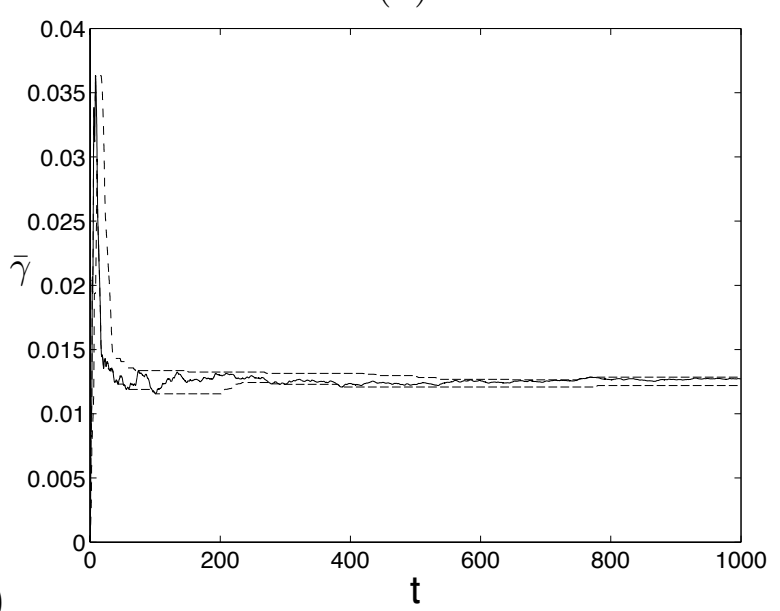

(c)

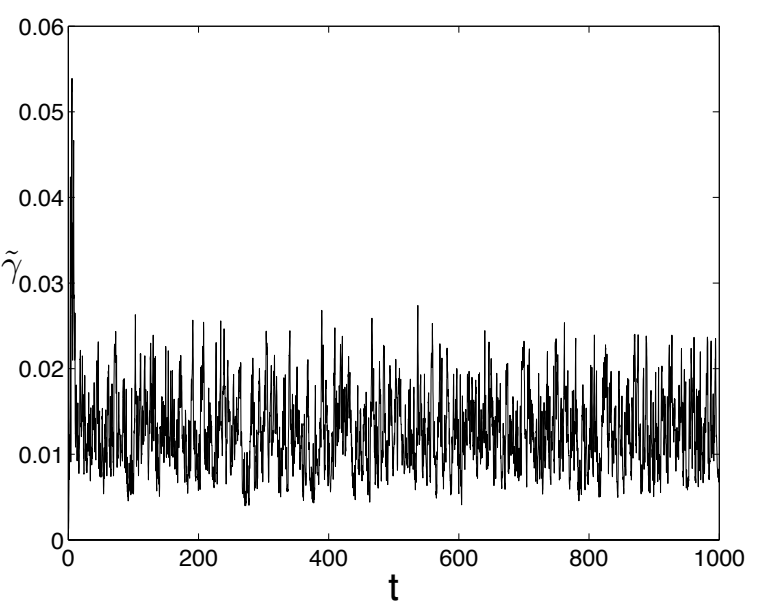

Fig. 3. Plots for $\left(\psi_{\text {slip }}, \sigma_{\mathrm{D}}\right)$ against time $t$ of (a) $V(t),(\mathrm{b}) \widetilde{\gamma}(t)$, and (c) $\bar{\gamma}(t)$ (solid), $\bar{\gamma}_{\min }(t)$ and $\bar{\gamma}_{\max }(t)$ (dashed).

impose a Dirichlet boundary condition. The oddness of the scalar field under the reflections $S_{1}$, $S_{2}$ in (3.6) is evident, with invariance under $R^{2}$, the half-turn rotation about the origin. The scalar field clearly tends to become well-mixed (red) within the square $\mathcal{D}$. On the boundary $\mathcal{C}$ there is a thin layer where the values rapidly tend to zero (green), together with cooler plumes (yellow) being entrained into the interior of the box. These boundary layers control the cooling of the fluid within, and so the decay rate of the scalar field.

To obtain an accurate measure of the decay rate we use longer runs, rescaling the scalar field to avoid underflow when required. Figure 3 shows the evolution of (a) $V(t)$ as a function of time, together with (b) the instantaneous decay rate $\widetilde{\gamma}(t)$ (measured between successive time-steps). There are strong random fluctuations and to obtain an accurate average decay rate, we take a running average over the window $[t / 2, t]$ to define

$$
\bar{\gamma}(t)=-t^{-1} \log [V(t) / V(t / 2)]
$$

The choice of expanding time window eliminates transient behaviour and averages over a greater part of the realisation as time increases. In a run up to time $t=T$ our best estimate of the scalar decay rate is $\bar{\gamma}(T)$ but to put a plausible and somewhat conservative error bar on 


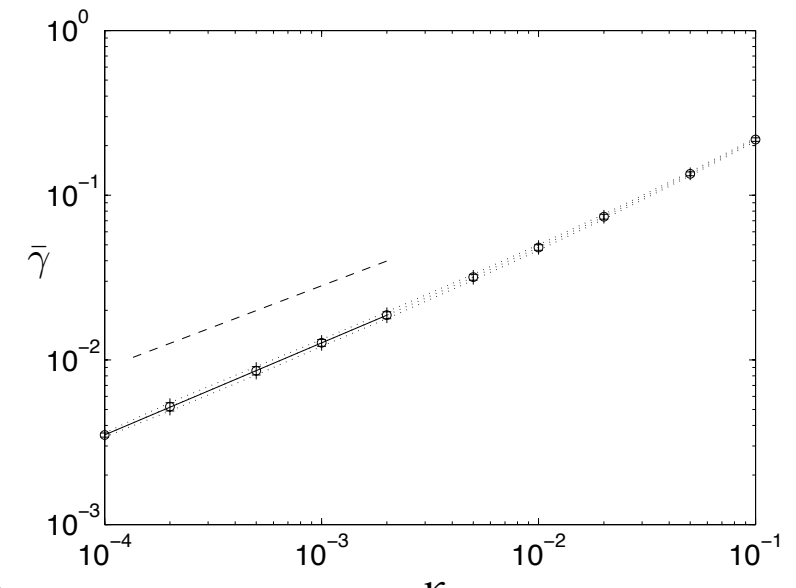

(a)

$\kappa$

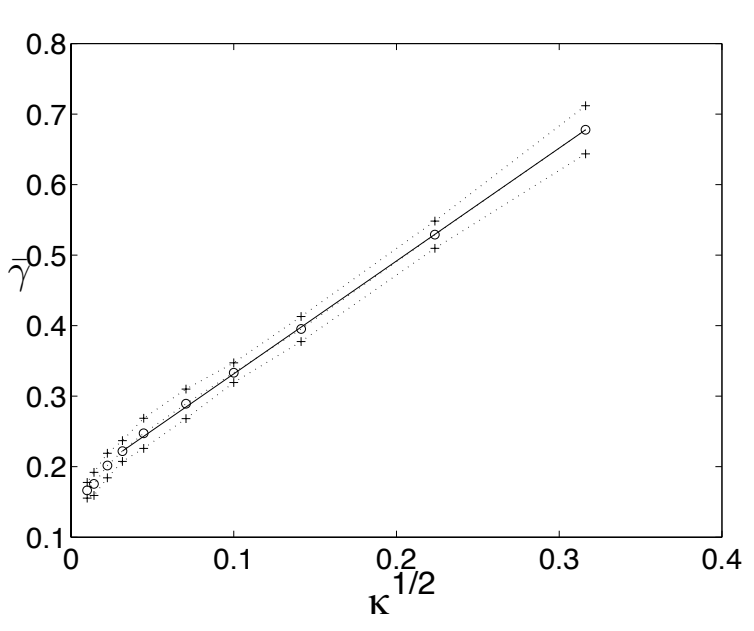

(b)

Fig. 4. Plots (a) for $\left(\psi_{\text {slip }}, \sigma_{\mathrm{D}}\right)$ showing $\bar{\gamma}$ (circles) and $\bar{\gamma} \pm \Delta \gamma$ (plus signs) against $\kappa$, and (b) for $\left(\psi_{\text {slip }}, \sigma_{\mathrm{N}}\right)$ showing $\bar{\gamma}$ (circles), and $\bar{\gamma} \pm \Delta \gamma$ (plus signs) against $\sqrt{\kappa}$. In (a) the solid line is a fit with slope 0.546 while the dashed line has slope 0.5 ; in (b) the solid line has slope 1.6.

this quantity we track upper and lower values of the average, defined by

$$
\bar{\gamma}_{\max }(t)=\max _{t / 2 \leq s \leq t} \bar{\gamma}(s), \quad \bar{\gamma}_{\min }(t)=\min _{t / 2 \leq s \leq t} \bar{\gamma}(s)
$$

In figure 3(c) $\bar{\gamma}(t)$ (solid), together with $\bar{\gamma}_{\min }(t)$ and $\bar{\gamma}_{\max }(t)$ (dashed) are shown. At the end of the run our final estimate of the decay rate and error bar is

$$
\gamma(\kappa) \simeq \bar{\gamma} \pm \Delta \gamma, \quad \bar{\gamma}=\bar{\gamma}(T), \quad \Delta \gamma=\bar{\gamma}_{\max }(T)-\bar{\gamma}_{\min }(T)
$$

Most runs of the random flows were taken to $T=1000$ to obtain the error bars shown, plotted in all graphs but often too small to be easily visible. However in the cases of slip flows with a Neumann boundary condition in a square or a plane layer (figures 4(b), 10(b)) the runs were taken to $T=2000$ to obtain better convergence of decay rates.

With a range of runs for different values of $\kappa$ we can seek the scaling behaviour of the decay rate as a function of $\kappa$ in the limit of small diffusivity,

$$
\gamma(\kappa) \simeq C \kappa^{\alpha} \quad(\kappa \rightarrow 0) .
$$

Figure 4(a) shows $\gamma(\kappa)$ (circles) plotted against $\kappa$ on a $\log -\log$ scale. Error bars are indicated (plus signs) but these are small and hardly visible. There is a clear straight-line scaling and the solid line fit has a slope of $\alpha \simeq 0.546$ : this is close to $\alpha=0.5$ and this slope is also indicated by a dashed line. There is clear evidence of scaling and our measurement of the exponent $\alpha$ is consistent with a value of $1 / 2$ as discussed in section 5 . To give a definite range of values of $\alpha$ from the data, we estimate the maximum and minimum slopes of straight lines that pass through the error bars in (3.9) for the range of $\kappa$ used for the fit (namely $10^{-4} \leq \kappa \leq 2 \times 10^{-3}$ ). This procedure yields $\alpha \in[0.527,0.562]$, although we note that there is some curvature seen in the data points, consistent with an overestimate of the limiting value as $\kappa \rightarrow 0$.

Having treated this case in some detail we now move more quickly. For the Neumann or noflux condition figures 5 and 4(b) are analogous to those for the Dirichlet case. However for the 
(a)

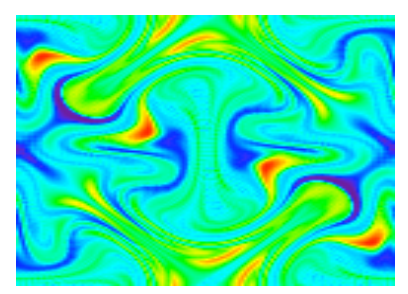

(b)

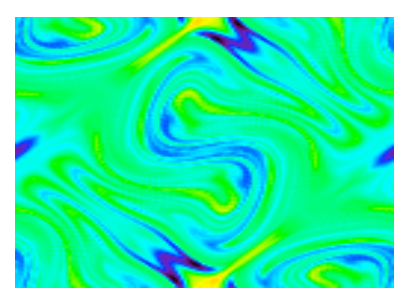

(c)

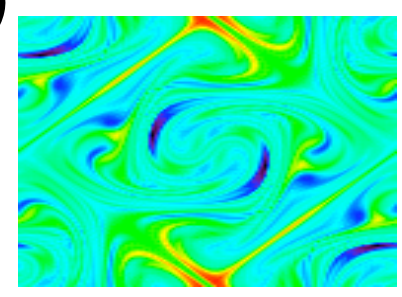

Fig. 5. Scalar field for $\left(\psi_{\text {slip }}, \sigma_{\mathrm{N}}\right)$ with $\kappa=0.001$ at (a) $t=10$, (b) 20 and (c) 30 .

Neumann boundary condition the scalar field decays much more rapidly and some care has to be taken, otherwise numerical error leads to pollution by the Dirichlet symmetry class and an incorrect measure of the decay rate. Recall that the field $\sigma$ transforms according to (3.6) under the reflections $S_{1}, S_{2}$ given in (2.16,2.17). From these equations we also obtain $R^{2} \sigma=\sigma$. Looking at the Fourier decomposition (2.6) we then have, with obvious notation,

$$
R^{2} \hat{\sigma}_{m, n}=\hat{\sigma}_{-m,-n}, \quad S_{1} \hat{\sigma}_{m, n}=(-1)^{m+n} \hat{\sigma}_{-n,-m}, \quad S_{2} \hat{\sigma}_{m, n}=(-1)^{m+n} \hat{\sigma}_{n, m} .
$$

Thus all the coefficients are real in view of (2.6) and for the two symmetry classes we have

$$
\hat{\sigma}_{m, n}=\hat{\sigma}_{-m,-n}=\mp(-1)^{m+n} \hat{\sigma}_{-n,-m}=\mp(-1)^{m+n} \hat{\sigma}_{n, m} \in \mathbb{R} .
$$

For the Neumann case (lower sign), the code was modified to impose these symmetries at occasional intervals, and so remove any parasitic Dirichlet solution.

Figure 5 shows typical results for a simulation. The Neumann boundary condition is evident in the evenness of the field under the reflection symmetries $S_{1}, S_{2}$. Except for this, though, there is no obvious role in the boundaries $\mathcal{C}$ in controlling the actual structure of the field, although $\boldsymbol{u} \cdot \boldsymbol{n}=0$ there (Salman \& Haynes, 2007). Even at the corners where $\boldsymbol{u}=0$, there are no obvious, persistent concentrations of scalar field. The decay may therefore be expected to be similar to the decay for a random flow without boundaries (see, e.g., Antonsen et al., 1996; Toussaint et al., 2000; Fereday \& Haynes, 2004; Salman \& Haynes, 2007) and indeed in figure $4(\mathrm{~b})$ we observe a long-time decay rate $\gamma(\kappa)$ (on a linear-square root scale) that appears to tend to a constant as $\kappa$ tends to zero, with a correction that appears to be of order $\sqrt{\kappa}$ (the data point for the smallest value of $\kappa$ is probably less accurate than the remaining ones). This data is consistent with an exponent $\alpha=0$. We note that decay on such a time-scale independent of $\kappa$ indicates the presence of diffusive structures of scale $\kappa^{1 / 2}$ in the scalar field, and so corrections to the decay rate with this $\kappa^{1 / 2}$ scaling are likely.

\subsection{Numerical simulations for the flow $\psi_{\text {no-slip }}$}

We now consider scalar decay in flows that satisfy a no-slip condition, $\boldsymbol{u}=0$ on $\mathcal{C}$; to do so we simply square the components $\phi_{m, n}$ in (2.12) that are used to build stream functions and initial conditions. The quadratic vanishing of the stream function along the boundary $\mathcal{C}$ ensures that the full no-slip condition is satisfied there. Similar to (3.4) we will use the random flow

$$
\psi_{\text {no-slip }}=\phi_{1,3}(x, y)^{2} \sin [\omega t+\chi(t)]+\phi_{3,1}(x, y)^{2} \cos [\omega t+\chi(t)] .
$$


(a)

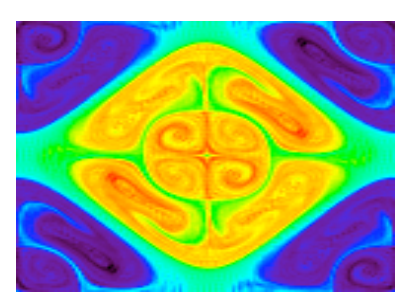

(d)

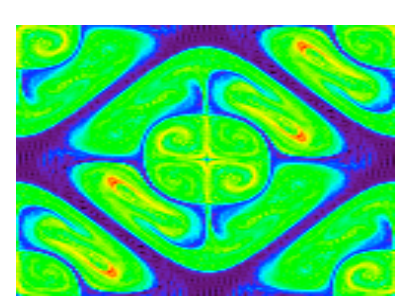

(b)

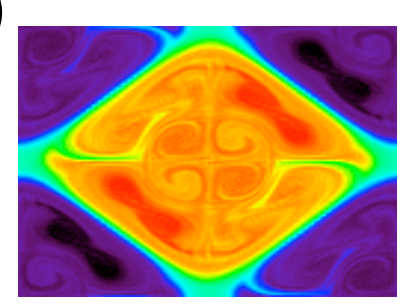

(e)

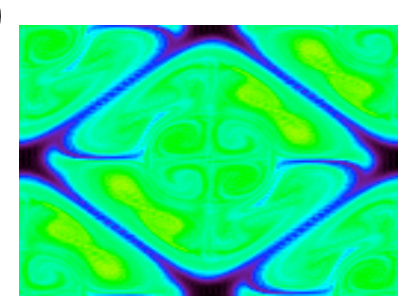

(c)

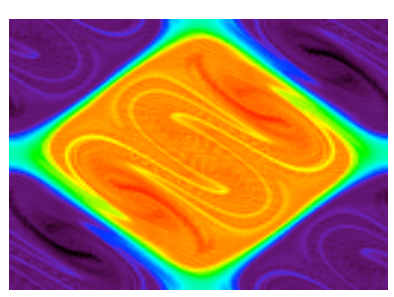

(f)

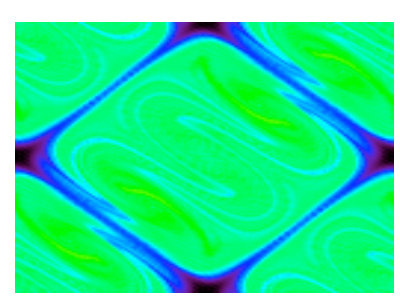

Fig. 6. Scalar field for $(\mathrm{a}-\mathrm{c})\left(\psi_{\text {no-slip }}, \sigma_{\mathrm{D}}\right)$ and $(\mathrm{d}-\mathrm{f})\left(\psi_{\text {no-slip }}, \sigma_{\mathrm{N}}\right)$ with $\kappa=0.001$. The time $t$ is $(\mathrm{a}, \mathrm{d})$ 5 , (b,e) 10 and $(\mathrm{c}, \mathrm{f}) 15$.

The stream function now does not change sign under reflections $(2.16,2.17)$ in the boundaries $\mathcal{C}$ and so (3.3) can no longer be satisfied. We have to discard these reflection symmetries in the no-slip case. Instead the relevant symmetries to consider are those that preserve orientation, in particular the translation $T$, the rotation $R^{2}$ about the origin, and the rotation $T R^{2}$ about $(\pi / 2, \pi / 2)$. We now have

$$
T, T R^{2}: \psi \rightarrow \psi, \quad \sigma \rightarrow \mp \sigma, \quad J \rightarrow J,
$$

with the upper sign holding for scalar evolution from $\sigma_{\mathrm{D}}$ and the lower sign from $\sigma_{\mathrm{N}}$. To illustrate this we give results of simulations starting with $\sigma_{\mathrm{D}}$ and $\sigma_{\mathrm{N}}$, shown in figure 6 . This figure can be compared with figures 2 and 5 .

It is important to note that because we no longer have reflectional symmetries $S_{1}$ and $S_{2}$ in the boundaries of the square domain $\mathcal{D}$, we can no long easily impose strict Dirichlet and Neumann boundary conditions on the scalar field. Instead the scalar field is odd or even respectively under the rotation through $\pi$ about the midpoint of each side, seen clearly in figure 6 . In fact we observe numerically in this and other figures that the Dirichlet and Neumann boundary conditions hold approximately, and so we will continue to use this terminology. Note that these boundary conditions are realistic for flows and scalars in a periodic cellular flow with corotating eddies separated by thin, thermally conducting no-slip boundaries (cf. Plunian et al. (1999) in a dynamo context), and so are not simply artificial. In addition, when we discuss theory in section 5, averaging over the random renewing flows to obtain an effective diffusivity will restore Dirichlet or Neumann boundary conditions to the averaged system.

In place of (3.11) we now have

$$
R^{2} \hat{\sigma}_{m, n}=\hat{\sigma}_{-m,-n}, \quad T \hat{\sigma}_{m, n}=(-1)^{m+n} \hat{\sigma}_{m, n}, \quad T R^{2} \hat{\sigma}_{m, n}=(-1)^{m+n} \hat{\sigma}_{-m,-n},
$$

and so fields satisfy

$$
\hat{\sigma}_{m, n}=\hat{\sigma}_{-m,-n}=\mp(-1)^{m+n} \hat{\sigma}_{m, n}=\mp(-1)^{m+n} \hat{\sigma}_{-m,-n} \in \mathbb{R},
$$




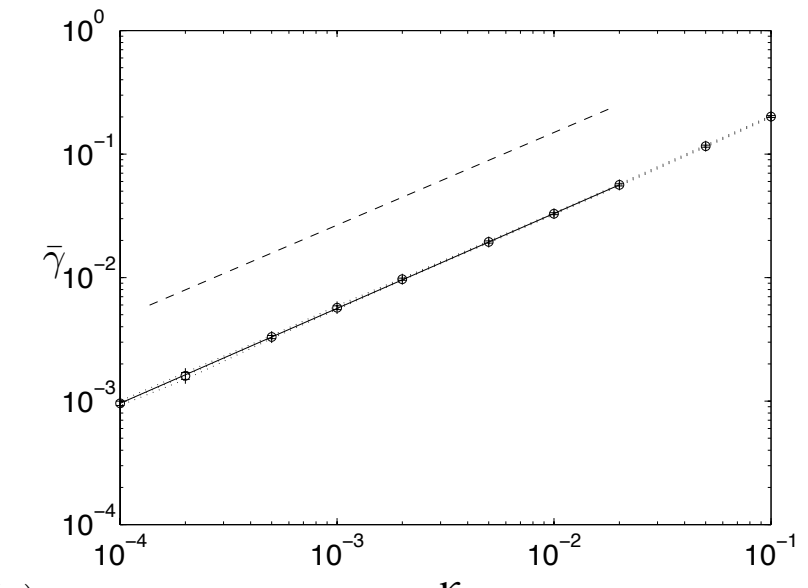

(a)$$
\kappa
$$

Fig. 7. Plot for (a) $\left(\psi_{\text {no-slip }}, \sigma_{\mathrm{D}}\right)$ and (b) $\left(\psi_{\text {no-slip }}, \sigma_{\mathrm{N}}\right)$ of $\bar{\gamma}$ (circles) and $\bar{\gamma} \pm \Delta \gamma$ (plus signs) against $\kappa$. The solid lines are fits with slope (a) 0.765 and (b) 0.625 . The dashed lines have slope 0.75 in (a) and 0.67 in (b).

starting with $\sigma_{\mathrm{D}}$ (upper sign) or $\sigma_{\mathrm{N}}$ (lower sign). Half the coefficients are identically zero in each case.

Turning now to the results of the simulations, for the Dirichlet case we see overall similar behaviour in the no-slip case (figure $6(\mathrm{a}-\mathrm{c})$ ) compared with the slip case (figure 2), except that no-slip boundary conditions appear to give thicker boundary layers, likely to slow down the loss of heat from the system. This slowing is evident in the scaling law for the decay rate $\gamma(\kappa)$ in figure 7(a) which shows a fall-off with exponent $\alpha$ measured as approximately 0.765 , close to a suggested theoretical value of $3 / 4$, as we shall discuss below. For the Neumann case, whereas for the slip boundary condition the boundary played no apparent role in the destruction of temperature fluctuations (figure 5) the long-time scalar distribution in figure $6(\mathrm{~d}-\mathrm{f}$ ) consists of a region of cool fluid (blue) in the relatively stagnant region near the boundary and a luke-warm interior (green). As argued by Lebedev \& Turitsyn (2004) these 'peripheral regions' control the mixing; however we find a larger exponent than the value $\alpha=1 / 2$ in their paper. Below in section 5 we will argue that it is $2 / 3$ in this case, with the corners now playing a controlling role. Note that in all the figures presented the colour scale (given in figure 2) is chosen with zero centred on green and the extremities of the scale (black or red) chosen to correspond to $\pm \max |\sigma|$. Thinking of the passive scalar field as temperature, in the plots in figure $6(\mathrm{a}-\mathrm{c})$, by symmetry there is fluid at both extremes of temperature whereas in $(\mathrm{d}-\mathrm{f})$ the scalar field is asymmetrical, there being very cold fluid near the boundary and no corresponding very hot fluid in the system.

\section{Simulations in a plane layer and Bloch wavenumbers}

Previous work by Salman \& Haynes (2007) focuses on evolution of passive scalars in an infinite plane layer. In this section we give some comparison with their work, and develop some further directions using Bloch wavenumbers. In our doubly periodic domain we take the plane layer to be the region, again called $\mathcal{D}$, with the lines $y-x= \pm \pi$ forming its boundary $\mathcal{C}$ depicted solid 


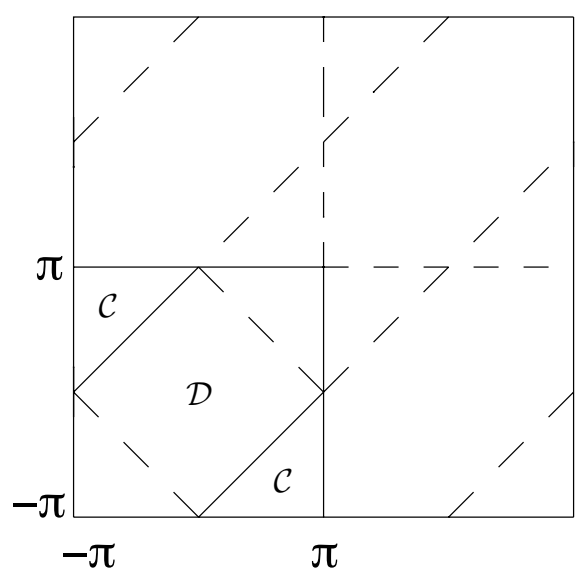

Fig. 8. Geometry of plane layer embedded in $2 \pi$-periodic space.

in figure 8: note how the plane layer continues periodically. To obtain a suitable flow all we need to do is to ensure that the flow breaks the previous symmetries that preserved the lines $x+y= \pm \pi$, while retaining the plane layer boundaries $y-x= \pm \pi$ as invariant. The symmetry group of the new, plane layer domain is reduced to 8 elements, generated by $\left\{R^{2}, T, R S\right\}$ and includes the reflections $S_{1}$ and $S_{2}$ defined in $(2.16,2.17)$.

For Neumann boundary conditions we may again take the $\sigma_{\mathrm{N}}$ given in (3.5) as initial condition. But for Dirichlet boundary conditions, in which the layers of fluid are alternately hot and cold, it is evident from figure 8 that we need to take an initial condition of greater periodicity than $2 \pi$, for example

$$
\sigma_{\frac{1}{2} \mathrm{D}}=\cos \frac{1}{2}(y-x) .
$$

This greater periodicity may easily be accommodated by redefining $\sigma$ in (4.2) to allow periodicity $4 \pi$ via the Bloch form

$$
\sigma(\boldsymbol{r}, t)=e^{i x / 2+i y / 2} \sum_{m, n} \hat{\sigma}_{m, n}(t) e^{i m x+i n y}, \quad \hat{\sigma}_{m, n}^{*}=\hat{\sigma}_{-m-1,-n-1} .
$$

In this case the initial condition (4.1) corresponds to setting $\hat{\sigma}_{-1,0}=\hat{\sigma}_{0,-1}=1 / 2$. (Note also that $V(t)$ in $(2.2)$ is now taken as the mean-square scalar over the $4 \pi$ periodic domain.)

\subsection{Numerical simulations for the flow $\psi_{\text {slip,pl }}$}

We set up the following flow which obeys a slip condition on the plane layer (subscript 'pl') boundaries,

$$
\psi_{\text {slip }, \mathrm{pl}}=\phi_{1,3}(x-c t, y-c t) \sin [\omega t+\chi(t)]+\phi_{3,1}(x+c t, y+c t) \cos [\omega t+\chi(t)] .
$$

The flow can now be thought of as travelling waves depicted in figure 1(c,d), travelling in the $\pm(1,1)$ directions and having their phase $\chi(t)$ reset randomly at each renewing time $t=j \tau$. Particles may now cross the lines $x+y= \pm \pi$ and this breaks the symmetry $S_{1}$ used earlier. We take $c=\omega=1$ in what follows and as usual $\tau=0.64$. Statistically, the flow is translationally invariant parallel to the line $y=x$. 
(a)

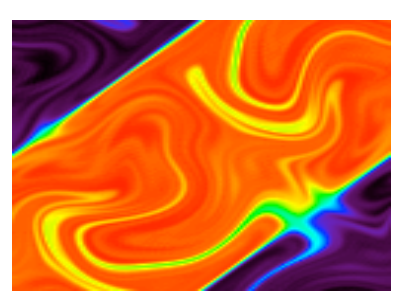

(d)

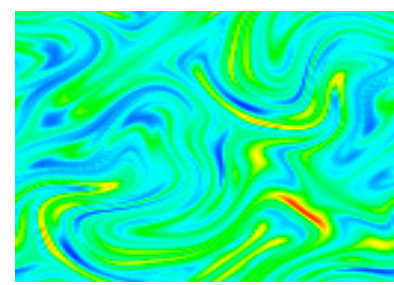

(b)

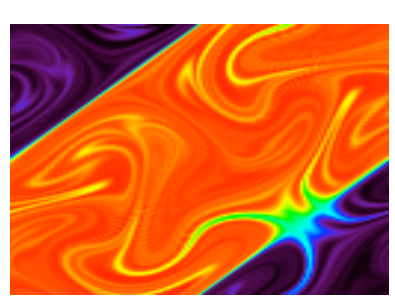

(e)

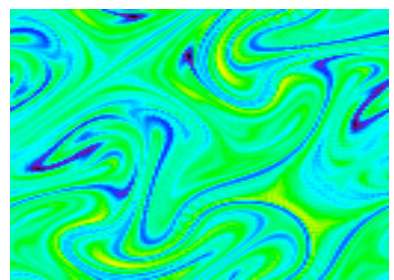

(c)

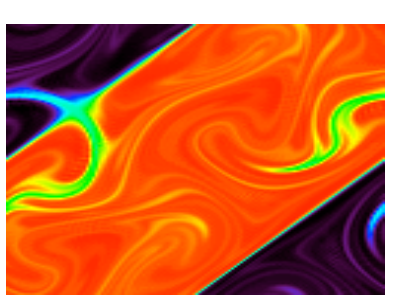

(f)

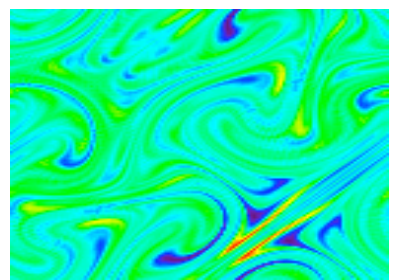

Fig. 9. Scalar field for $(\mathrm{a}-\mathrm{c})\left(\psi_{\mathrm{slip}, \mathrm{pl}}, \sigma_{\frac{1}{2} \mathrm{D}}\right)$ and $(\mathrm{d}-\mathrm{f})\left(\psi_{\text {slip,pl }}, \sigma_{\mathrm{N}}\right)$ with $\kappa=0.001$ at $(\mathrm{a}, \mathrm{d}) t=100$, (b,e) 200 and $(c, f) 300$.

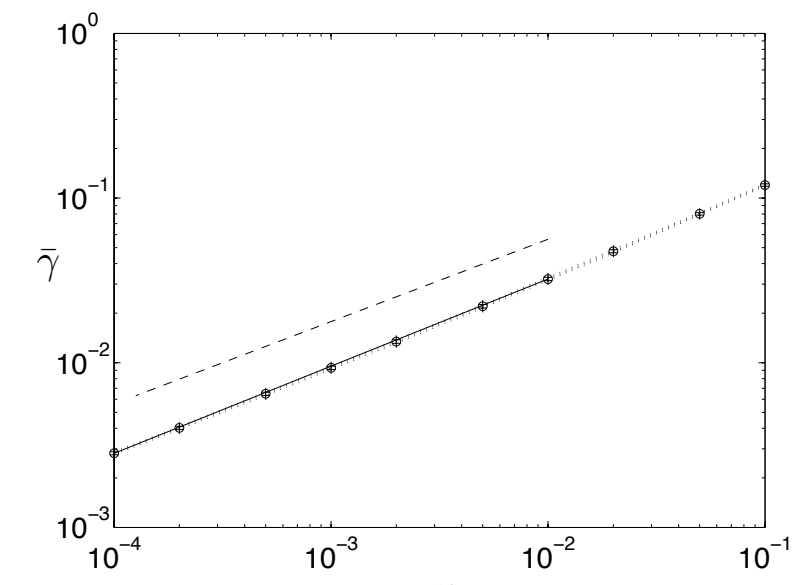

(a)

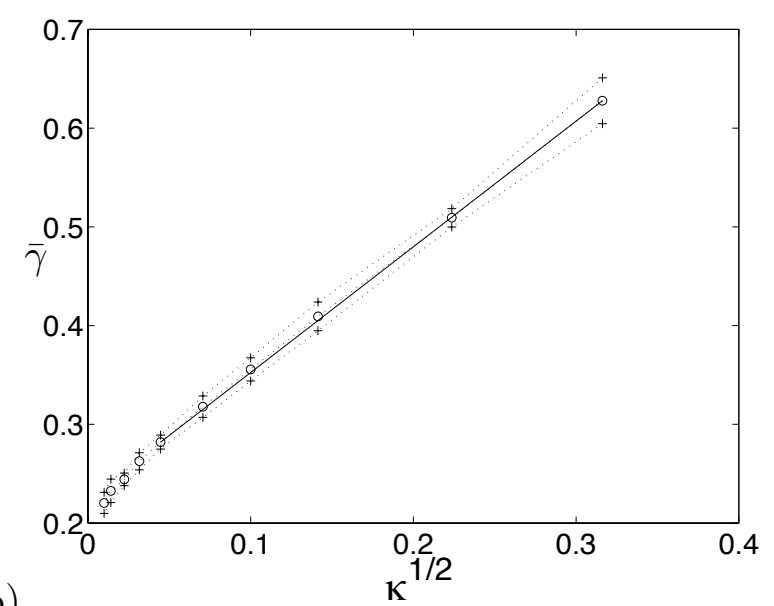

(b)

Fig. 10. Plots (a) for $\left(\psi_{\text {slip,pl }}, \sigma_{\frac{1}{2} \mathrm{D}}\right)$ showing $\bar{\gamma}$ (circles) and $\bar{\gamma} \pm \Delta \gamma$ (plus signs) against $\kappa$, and (b) for $\left(\psi_{\text {slip,pl }}, \sigma_{\mathrm{N}}\right)$ showing $\bar{\gamma}$ (circles), and $\bar{\gamma} \pm \Delta \gamma$ (plus signs) against $\sqrt{\kappa}$. The solid lines are fits with slopes (a) 0.53 and (b) 1.27, while in (a) the dashed line has slope 0.5 .

Figure 9 shows runs for $\psi_{\text {slip,pl }}$ with $\sigma_{\frac{1}{2} \mathrm{D}}$ and $\sigma_{\mathrm{N}}$ : the only symmetry left in these fields is that of being odd or even under the reflection $S_{2}$ in (2.17). For the Dirichlet case we now have for the coefficients

$$
\hat{\sigma}_{m, n}=-(-1)^{m+n} \hat{\sigma}_{n, m}=\hat{\sigma}_{-m-1,-n-1}^{*}=-(-1)^{m+n} \hat{\sigma}_{-n-1,-m-1}^{*},
$$

(incorporating (4.2)) while in the Neumann case they are governed by

$$
\hat{\sigma}_{m, n}=(-1)^{m+n} \hat{\sigma}_{n, m}=\hat{\sigma}_{-m,-n}^{*}=(-1)^{m+n} \hat{\sigma}_{-n,-m}^{*}
$$

(incorporating (2.6)). Figure 10 shows decay rates with exponent $\alpha \simeq 1 / 2$ in the Dirichlet case and zero in the Neumann case: in the latter case again the boundaries appear to play no 
(a)

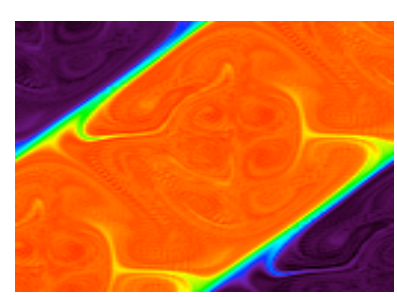

(d)

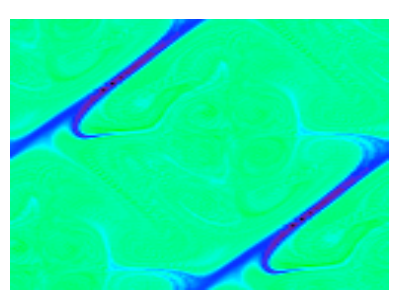

(b)

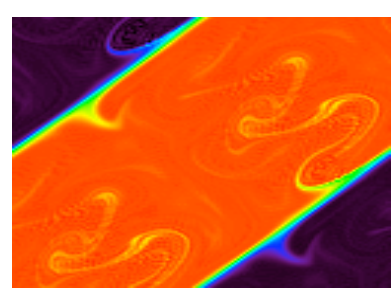

(e)

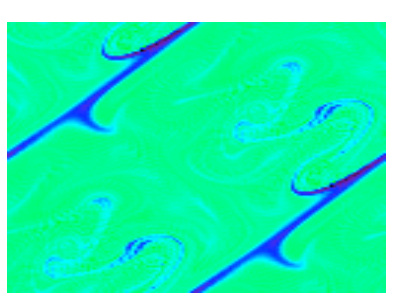

(c)

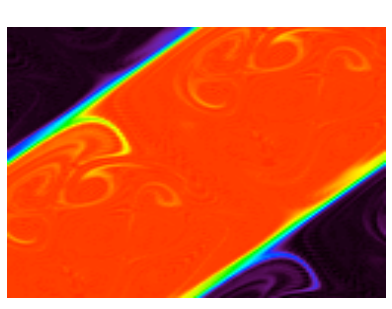

(f)

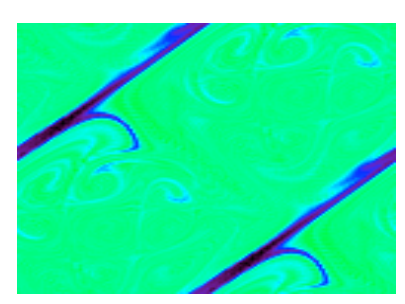

Fig. 11. Scalar field for $(\mathrm{a}-\mathrm{c})\left(\psi_{\text {no-slip,pl }}, \sigma_{\frac{1}{2} \mathrm{D}}\right)$ and $(\mathrm{d}-\mathrm{f})\left(\psi_{\text {no-slip,pl }}, \sigma_{\mathrm{N}}\right)$ with $\kappa=0.001$ at $(\mathrm{a}, \mathrm{d}) t=10$, $(\mathrm{b}, \mathrm{e}) 20$ and $(\mathrm{c}, \mathrm{f}) 30$.

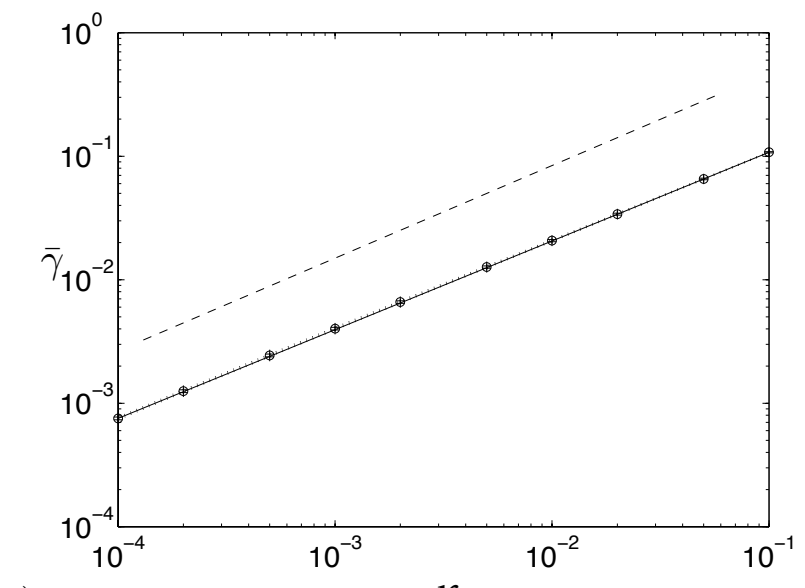

(a)

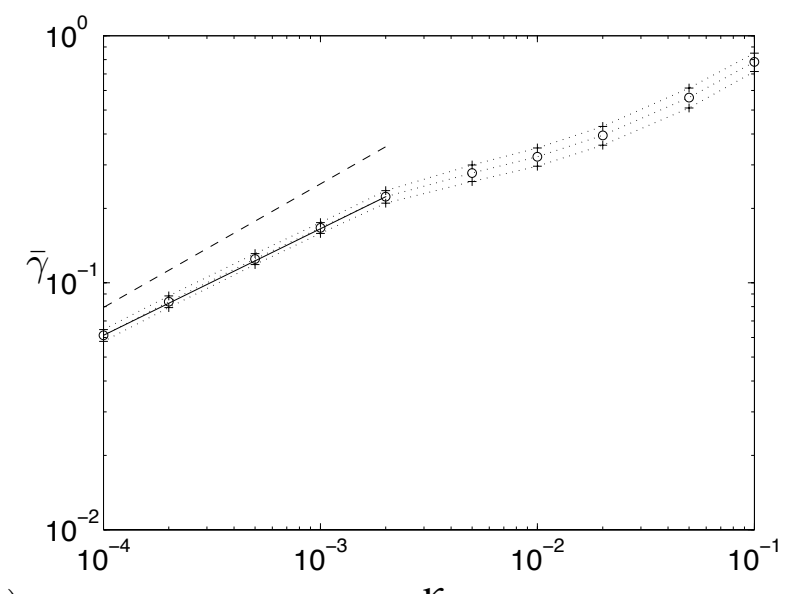

(b)

Fig. 12. Plot for (a) $\left(\psi_{\text {no-slip,pl }}, \sigma_{\frac{1}{2} \mathrm{D}}\right)$ and (b) $\left(\psi_{\text {no-slip,pl }}, \sigma_{\mathrm{N}}\right)$ of $\bar{\gamma}$ (circles) and $\bar{\gamma} \pm \Delta \gamma$ (plus signs) against $\kappa$ for the case. The solid line is a fit with slope (a) 0.718 , (b) 0.43 while the dashed line has slope 0.75 in (a) and 0.5 in (b).

particular role in the mixing and destruction of fluctuations.

\subsection{Numerical simulations for the flow $\psi_{\text {no-slip,pl }}$}

We now reach the problem studied in Salman \& Haynes (2007), of flow in a plane layer with a no-slip condition and a scalar Neumann boundary condition. We consider the following random flow which obeys a no-slip condition and is statistically translationally invariant,

$$
\psi_{\text {no-slip,pl }}=\phi_{1,3}(x-c t, y-c t)^{2} \sin [\omega t+\chi(t)]+\phi_{3,1}(x+c t, y+c t)^{2} \cos [\omega t+\chi(t)] .
$$

Figure 11 shows the corresponding scalar fields with Dirichlet and Neumann conditions and figure 12 measurements of the scaling exponents. For both Dirichlet and Neumann conditions 
the scalar field maps to itself only under the translation $T$. For the Dirichlet conditions we again obtain an exponent $\alpha \simeq 3 / 4$, as for the case of no-slip flow in a square domain. For Neumann boundary conditions the exponent is $\alpha \simeq 1 / 2$ in keeping with Lebedev \& Turitsyn (2004), although convergence is rather slow and the slope poorly determined; we offer further comparison with theory in section 5 below.

\subsection{Mixed Dirichlet/Neumann boundary conditions for flow in a square domain}

Finally, for our simulations of random flows, we note that with the Bloch wavenumber form (4.2) of the scalar field we may also consider flows in the square domain of section 3 in the case of Dirichlet boundary conditions on the lines $y-x= \pm \pi$ and Neumann boundary conditions on the lines $x+y= \pm \pi$, simply by taking the initial condition $\sigma_{\frac{1}{2} \mathrm{D}}$ in (4.1). Results for the flows $\psi_{\text {slip }}$ and $\psi_{\text {no-slip }}$ are shown in figures 13 and 14. For $\psi_{\text {slip }}$ the scalar field satisfies

$$
\sigma=S_{1} \sigma=-S_{2} \sigma=R^{2} \sigma
$$

and Fourier coefficients obey

$$
\hat{\sigma}_{m, n}=-(-1)^{m+n} \hat{\sigma}_{-n-1,-m-1}=-(-1)^{m+n} \hat{\sigma}_{n, m}=\hat{\sigma}_{-m-1,-n-1} .
$$

Similarly for $\psi_{\text {no-slip }}$ we have

$$
\sigma=R^{2} \sigma=T \sigma=T R^{2} \sigma
$$

and

$$
\hat{\sigma}_{m, n}=\hat{\sigma}_{-m-1,-n-1}=-(-1)^{m+n} \hat{\sigma}_{-m-1,-n-1}=-(-1)^{m+n} \hat{\sigma}_{m, n} .
$$

In addition (4.2) holds in both cases. We again obtain exponents of $\alpha \simeq 1 / 2$ and $\alpha \simeq 3 / 4$ for the decay of scalar in the square domain: the Neumann boundary condition and the corners appear to play no particular role. There are clearly stagnant regions of fluid near the corners, but it is the large gradients along the boundaries where the Dirichlet boundary condition is applied that control the decay of the passive scalar in the square domain.

\section{$5 \quad$ Scaling laws}

We can summarise our results for the random flows above in table 5. Here we have given suggested 'analytical' values of exponent $\alpha$ in the last column, and we now present justification for these.

\subsection{Theory for decay in a no-slip flow with Neumann boundary conditions}

Our starting point is the theory used by Lebedev \& Turitsyn (2004) and Salman \& Haynes (2007) who give the value $\alpha=1 / 2$ for no-slip flow with Neumann boundary conditions, corresponding to line (viii) of the table. As this theory provides the key to understanding all of our results, we first need briefly to rederive the formulae appropriate to the family of random 
(a)

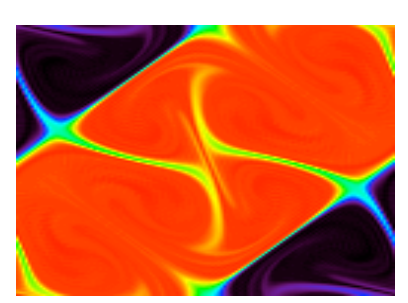

(d)

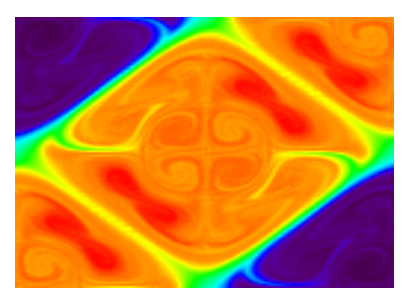

(b)

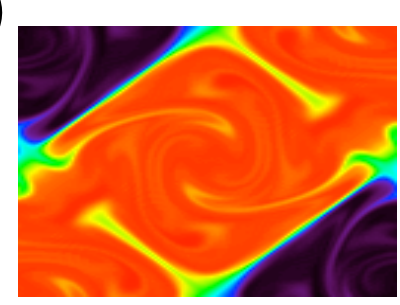

(e)

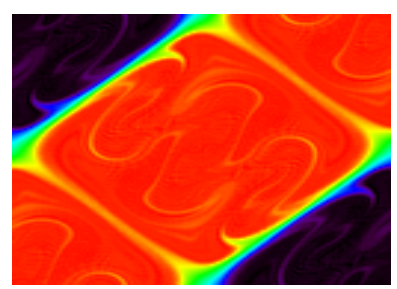

(c)

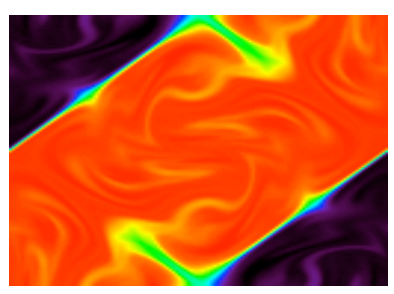

(f)

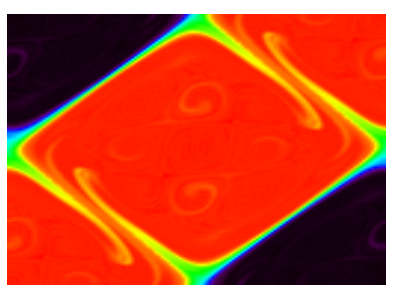

Fig. 13. Scalar field for $(\mathrm{a}-\mathrm{c})\left(\psi_{\text {slip }}, \sigma_{\frac{1}{2} \mathrm{D}}\right)$, (d-f) $\left(\psi_{\text {no-slip }}, \sigma_{\frac{1}{2} \mathrm{D}}\right)$ with $\kappa=0.001$ at (a) $t=25$, (b) 50, (c) 75, (d) $t=10$, (e) 20 and (f) 30 .

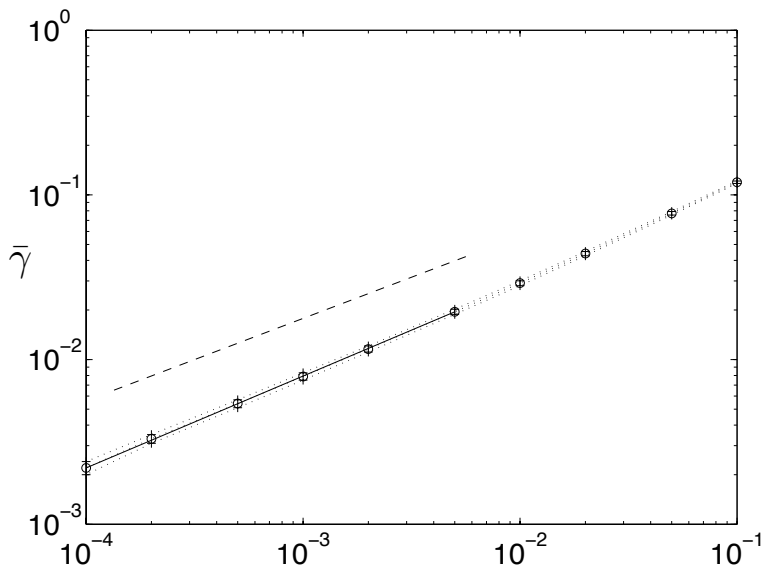

(a)

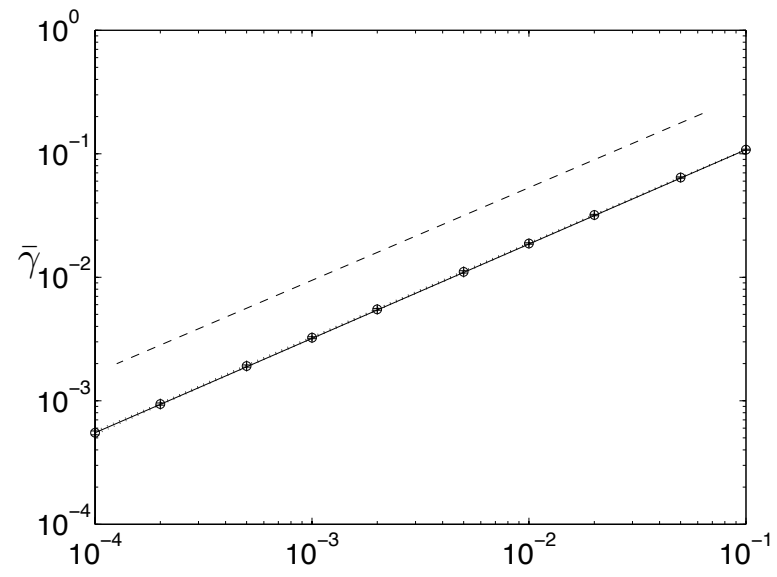

(b)

$\kappa$

Fig. 14. (a) Plot for (a) $\left(\psi_{\text {slip }}, \sigma_{\frac{1}{2} \mathrm{D}}\right)$ and (b) $\left(\psi_{\text {no-slip }}, \sigma_{\frac{1}{2} \mathrm{D}}\right)$ of $\bar{\gamma}$ (circles) and $\bar{\gamma} \pm \Delta \gamma$ (plus signs) against $\kappa$. The solid line is a fit with slope (a) 0.553, (b) 0.762 and the dashed line has slope (a) 0.5, (b) 0.75.

renewing flows used. All the results in this subsection are derived in the above papers, which may be consulted for more discussion

The random flows used are chosen to be independent and identically distributed on each time interval $j \tau \leq t \leq(j+1) \tau$. If we use a Taylor series expansion for the scalar field $\sigma(j \tau+t)$ in terms of the field $\sigma(j \tau)$ we obtain

$$
\sigma(j \tau+t)=\sigma(j \tau)+t\left(-\boldsymbol{u} \cdot \nabla+\kappa \nabla^{2}\right) \sigma(j \tau)+\frac{1}{2} t^{2}\left(-\boldsymbol{u} \cdot \nabla+\kappa \nabla^{2}\right)^{2} \sigma(j \tau)+\cdots
$$

We have for convenience taken the flow $\boldsymbol{u}$ that operates over the time interval to be steady but this condition may easily be relaxed. If we now average over the family of flows and take 


\begin{tabular}{|c|c|c|c|c|c|c|}
\hline & domain & flow & initial scalar & $\omega$ & measured $\alpha$ & analytical $\alpha$ \\
\hline$(i)$ & square & $\psi_{\text {slip }}$ & $\sigma_{\mathrm{D}}$ & 1 & 0.546 & $1 / 2$ \\
$($ ii $)$ & square & $\psi_{\text {slip }}$ & $\sigma_{\mathrm{N}}$ & 1 & 0 & 0 \\
$($ iii $)$ & square & $\psi_{\text {no-slip }}$ & $\sigma_{\mathrm{D}}$ & 1 & 0.765 & $3 / 4$ \\
$($ iv $)$ & square & $\psi_{\text {no-slip }}$ & $\sigma_{\mathrm{N}}$ & 1 & 0.625 & $2 / 3$ \\
\hline$(v)$ & layer & $\psi_{\text {slip,pl }}$ & $\sigma_{\frac{1}{2} \mathrm{D}}$ & 1 & 0.53 & $1 / 2$ \\
$(v i)$ & layer & $\psi_{\text {slip,pl }}$ & $\sigma_{\mathrm{N}}$ & 1 & 0 & 0 \\
$($ vii $)$ & layer & $\psi_{\text {no-slip,pl }}$ & $\sigma_{\frac{1}{2} \mathrm{D}}$ & 1 & 0.718 & $3 / 4$ \\
$($ viii $)$ & layer & $\psi_{\text {no-slip,pl }}$ & $\sigma_{\mathrm{N}}$ & 1 & 0.43 & $1 / 2$ \\
\hline (ix $)$ & square & $\psi_{\text {slip }}$ & $\sigma_{\frac{1}{2} \mathrm{D}}$ & 1 & 0.553 & $1 / 2$ \\
$(x)$ & square & $\psi_{\text {no-slip }}$ & $\sigma_{\frac{1}{2} \mathrm{D}}$ & 1 & 0.762 & $3 / 4$ \\
\hline
\end{tabular}

Table 1

Summary of results: random flows.

them to have the property that $\langle\boldsymbol{u}\rangle=0$ we obtain, with $t=\tau$,

$$
\begin{aligned}
\bar{\sigma}((j+1) \tau) & =\bar{\sigma}(j \tau)+\tau \kappa \nabla^{2} \bar{\sigma}(j \tau)+\frac{1}{2} \tau^{2}\left\langle(\boldsymbol{u} \cdot \nabla)^{2}\right\rangle \bar{\sigma}(j \tau)+\cdots \\
& =\bar{\sigma}(j \tau)+\tau \kappa \nabla^{2} \bar{\sigma}(j \tau)+\frac{1}{2} \tau^{2} \frac{\partial}{\partial x_{j}}\left(\left\langle u_{j} u_{k}\right\rangle \frac{\partial}{\partial x_{k}} \bar{\sigma}(j \tau)\right)+\cdots
\end{aligned}
$$

Here we have retained just the leading order effect of the flow and the leading order effect of diffusion, and $\bar{\sigma}$ denotes the ensemble-averaged scalar field. Crucially, we have used the independence of the flow $\boldsymbol{u}$ for the time interval $j \tau \leq t \leq(j+1) \tau$ and the scalar field $\bar{\sigma}(j \tau)$ at the beginning of this interval. This independence allows us to write, for example, $\langle\boldsymbol{u}(t) \boldsymbol{u}(t) \sigma(j \tau)\rangle=\langle\boldsymbol{u}(t) \boldsymbol{u}(t)\rangle \bar{\sigma}(j \tau)$ and make analytical progress. If we now consider the evolution of the scalar field over time-scales much longer than $\tau$, then (5.2) amounts to a finite difference approximation to a diffusion equation,

$$
\frac{\partial \bar{\sigma}}{\partial t}=\frac{\partial}{\partial x_{j}}\left(D_{j k} \frac{\partial \bar{\sigma}}{\partial x_{k}}\right)+\kappa \nabla^{2} \bar{\sigma}
$$

where the diffusivity tensor is ${ }^{1}$

$$
D_{j k}=\frac{1}{2} \tau\left\langle u_{j} u_{k}\right\rangle
$$

and is generally a function of both spatial coordinates.

$\overline{1}$ Note there is an extra factor of $\frac{1}{2}$ compared with the calculation in equation (36) of Salman \& Haynes (2007). No such factor is present in the equivalent, integral formula for a stationary random flow given in equation (1.5) of Lebedev \& Turitsyn (2004), and appears because our flows are not stationary. It can be thought of as arising in our case since starting from a randomly chosen starting time $t$, the steady flow at that time continues to act for a time $\tau / 2$ on average. 
Now although we have obtained this result using an expansion in powers of a correlation time $\tau$, it is in fact an expansion in powers of $\tau \boldsymbol{u}$. The key observation of Lebedev \& Turitsyn (2004) is that near a no-slip boundary $\boldsymbol{u} \rightarrow 0$ and so the approximation becomes good near to that boundary regardless of whether the correlation time $\tau$ for the bulk of the flow is small or not. Thus the results obtained for no-slip boundary layers forced by random flows have a universal nature. They argue that if a no-slip boundary is given by $x=0$, say, then the components of the diffusivity tensor will scale with the form

$$
D_{x x} \simeq y^{2} f_{x x}(x), \quad D_{x y} \simeq y^{3} f_{x y}(x), \quad D_{y y} \simeq y^{4} f_{y y}(x) \quad(y \rightarrow 0) .
$$

(with the obvious notation $\left.\left(x_{1}, x_{2}\right)=(x, y)\right) .{ }^{2}$ In a situation in which the layer has a thickness controlled by diffusion, as is the case in the final exponential decay regime we are studying, the final two terms in (5.3) balance and this leads to the scalar field variation being concentrated in a boundary layer with scales

$$
x=O(1), \quad y=O\left(\kappa^{1 / 4}\right) \quad(\kappa \rightarrow 0) .
$$

With these scales, none of the diffusive terms on the right-hand side of (5.3) may be neglected. In the case of Neumann boundary conditions, it is now the slow decay of the scalar field in the boundary layer that is the source of fluctuations in the interior (where they mix rapidly on the advective time scale), and this decay is measured in the final regime. Bringing, then, the first, time derivative term in (5.3) into the balance requires that the decay rate scale as $\gamma=O\left(\kappa^{1 / 2}\right)$ and so the corresponding value is $\alpha=1 / 2$ as in line (viii) of the table (Lebedev \& Turitsyn, 2004).

This is a general argument, also valid as a local picture if the boundaries are curved. In a plane layer, if in addition the flow is statistically independent of the coordinate $x$ along the layer then so also are $D_{i j}$ and $\bar{\sigma}$, and only the term involving $D_{y y}$ is relevant. In the final, decay regime it is the $y^{4}$ behaviour of $D_{y y}$ near the boundary that is important, and the equation (5.3) collapses to

$$
\frac{\partial \bar{\sigma}}{\partial t}=\frac{\partial}{\partial y}\left(\mu y^{4} \frac{\partial \bar{\sigma}}{\partial y}\right)+\kappa \frac{\partial^{2} \bar{\sigma}}{\partial y^{2}}
$$

where $D_{y y} \simeq \mu y^{4}$ for small $y$. Solving this numerically for exponential decay as an eigenvalue problem with a no-flux boundary condition $(\partial \bar{\sigma} / \partial y=0$ at $y=0)$ gives the decay rate in this case as

$$
\gamma=C(\mu \kappa)^{1 / 2}, \quad C \simeq 1.81
$$

(Lebedev \& Turitsyn, 2004). This result has been tested on the no-slip flow $\psi_{\text {no-slip,pl }}$ in the plane layer given by (4.6) for which $\mu=45 \tau / 2 \simeq 14.4$. Figure 15 (a) shows good convergence consistent with the theoretical result, in view of the $\kappa^{1 / 4}$ corrections that would be present in the theory and the values of $\kappa$ down to only $10^{-4}$ in these simulations.

$\overline{2}$ For the present discussion the axes are rotated and translated with respect to the ones we have been using in earlier sections for our simulations. In (5.5) $x$ points along a boundary layer and $y$ is perpendicular; the meaning should be clear from the context. 


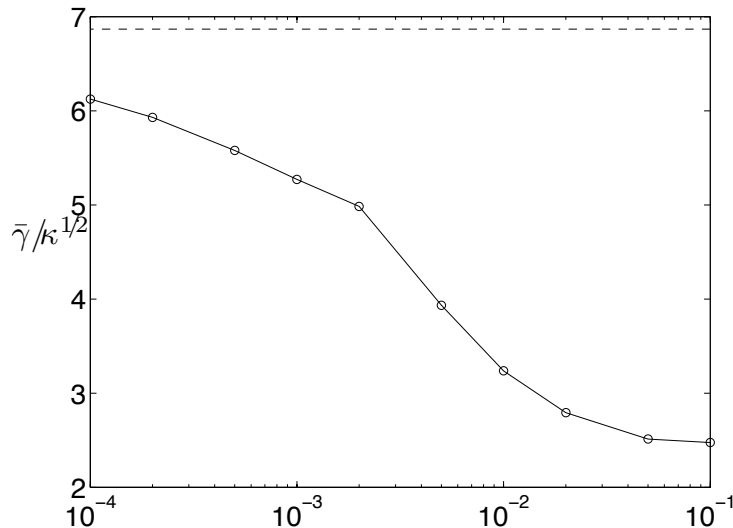

(a)

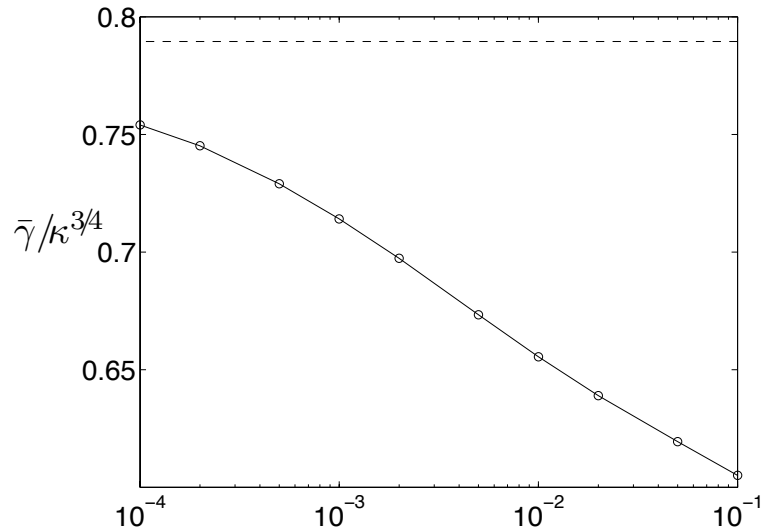

(b)
K

(c)

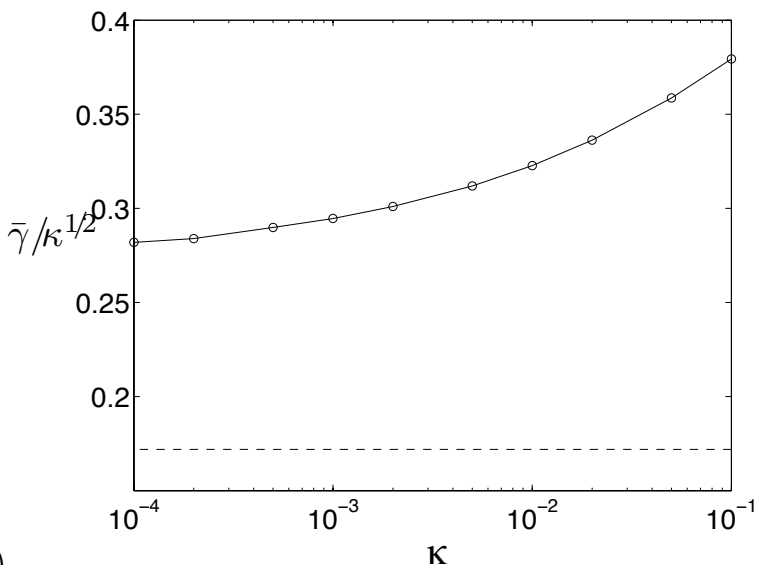

$\kappa$

Fig. 15. Plot of scaled decay rate (a) $\kappa^{-1 / 2} \gamma(\kappa)$ against $\kappa$ for no-slip flow $\psi_{\text {no-slip,pl }}$, Neumann boundary

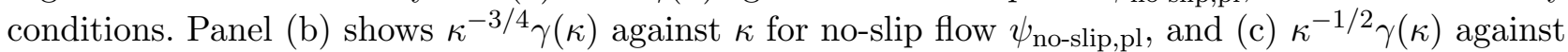
$\kappa$ for slip flow $\psi_{\text {slip,pl }}$, both with Dirichlet boundary conditions. In each case numerical results are given by markers (solid lines) and theoretical results from (5.8), (5.11) and (5.14) are dashed.

\subsection{Theory for decay in other configurations}

As indicated in Lebedev \& Turitsyn (2004), for a no-slip flow containing a scalar satisfying Dirichlet boundary conditions, equation (5.3) remains valid. However now the time derivative term on the left-hand side drops out as the decay rate $\gamma=O\left(\kappa^{3 / 4}\right)$ and so this term is asymptotically smaller than the terms on the right-hand side. The scalar distribution in the boundary layer is quasi-static. The slow decay of the scalar in a square or plane layer domain is controlled by the loss of scalar through the boundary layer, which may be calculated using

$$
\frac{\partial}{\partial t} \int_{\mathcal{D}} \bar{\sigma} d S=\kappa \int_{\mathcal{C}} \boldsymbol{n} \cdot \nabla \bar{\sigma} d s
$$

Since $\boldsymbol{n} \cdot \nabla \bar{\sigma}=O\left(\kappa^{-1 / 4}\right) \bar{\sigma}$ the decay rate is calculated to scale as $\gamma=O\left(\kappa^{3 / 4}\right)$, confirming the scaling results given in lines (iii, vii, $x$ ) in the table. Note that the boundary layers thicken near the corners (as we shall discuss below) but they make a negligible contribution to the integral. In the case where the domain is a plane layer, so that translational invariance along the 
boundary holds, then equation (5.7) remains valid, except that the time-derivative is now asymptotically negligible as $\kappa \rightarrow 0$. As pointed out by Lebedev \& Turitsyn (2004), this may be integrated to yield

$$
\bar{\sigma}=\bar{\sigma}_{\infty}(t) F\left[y(\mu / \kappa)^{1 / 4}\right], \quad F(s)=\frac{2 \sqrt{2}}{\pi} \int_{0}^{s} \frac{d r}{r^{4}+1}
$$

where $\bar{\sigma}_{\infty}(t)$ is the value of the (well-mixed) scalar outside the boundary layer $y \gg \kappa^{1 / 4}$. Applying now (5.9) gives the decay rate for a layer of depth $L$ as

$$
\gamma(\kappa)=\frac{4 \sqrt{2}}{\pi L} \mu^{1 / 4} \kappa^{3 / 4}
$$

For the no-slip flow $\psi_{\text {no-slip,pl }}$ in (4.6) we have $\mu=45 \tau / 2 \simeq 14.4, L=\pi \sqrt{2}$ and figure 15 (b) shows good agreement between theory and simulation, particularly noting the scale on the vertical axis.

We now turn to the slip flows. In the case of Neumann boundary conditions, it is clear that the boundaries do not play a controlling role in the decay of the scalar field (Salman \& Haynes, 2007): there is no evidence of boundary layers in figures $5,9(\mathrm{~d}-\mathrm{f})$ for example. In a short correlation time limit, the diffusivity tensor near the boundary will have components

$$
D_{x x} \simeq f_{x x}(x), \quad D_{x y} \simeq y f_{x y}(x), \quad D_{y y} \simeq y^{2} f_{y y}(x) \quad(y \rightarrow 0) .
$$

These dependences would give a boundary layer scaling of $y=O\left(\kappa^{1 / 2}\right)$ and a corresponding time scale in the boundary layer equation (5.3) of $\gamma=O(1)$. These spatial and temporal scales are the same as for the destruction of scalar fluctuations in the body of the fluid. So for Neumann boundary conditions, there is in fact no boundary layer present, that is no separation of space or time scales. The decay rate has $\alpha=0$ and we expect $\gamma=\gamma_{0}+O(\sqrt{\kappa})$ as found numerically, confirming lines $(i i, v i)$ of the table.

The situation is different for the Dirichlet boundary condition, because with scales $y=O\left(\kappa^{1 / 2}\right)$ in the boundary layer, relationship (5.9) now implies that the total scalar in the domain will decay at a rate $\gamma=O\left(\kappa^{1 / 2}\right)$ as $\kappa \rightarrow 0$, confirming lines $(i, v, i x)$ of the table. The scalar again mixes on an advective time scale in the cell, and its flux out is controlled by the boundary layers. In the case of a layer that is statistically translationally invariant in $x$ we obtain $D_{y} \simeq \mu y^{2}$. Integrating the boundary layer equation gives

$$
\bar{\sigma}=\bar{\sigma}_{\infty}(t) \frac{2}{\pi} \tan ^{-1}\left(y \sqrt{\frac{\mu}{\kappa}}\right)
$$

and a decay rate

$$
\gamma(\kappa)=\frac{2}{\pi L} \mu^{1 / 2} \kappa^{1 / 2}
$$

For the flow $\psi_{\text {slip,pl }}$ in (4.3) this result is checked in figure $15(\mathrm{c})$, the value of $\mu$ being $\mu=$ $9 \tau / 4 \simeq 1.44$. There is rapid convergence of $\kappa^{-1 / 2} \gamma(\kappa)$, but to a value that seems around twice the theoretical one. The reason for this disagreement in the actual value, but not the scaling, is because the correlation time $\tau$ is not sufficiently small for the approximation to be valid for 
this flow. Whereas the result $(5.3,5.4)$ will hold for any random no-slip flow because $\boldsymbol{u} \tau \rightarrow 0$ as the no-slip boundary is approached, the corresponding theory leading to (5.14) for a slip flow only holds if the correlation time $\tau \rightarrow 0$ since $\boldsymbol{u}$ does not tend to zero on the boundary, only its normal component. Further simulations, which we do not report here, show that agreement improves provided that both $\tau$ and $\kappa$ tend to zero (Zaggout, 2012).

The only remaining line of the table to be understood is line (iv): a Neumann boundary condition for no-slip flow in a square. Here there is a slower decay than is the case for a plane layer and examination of figure $6(\mathrm{~d}-\mathrm{f})$ reveals why. The corners of the square act as reservoirs of scalar that is slowly released into the body of the fluid, and this controls the time-scale of homogenisation in the domain. For the flows we consider we will have the following scaling for the diffusivity tensor as the corners are approached,

$$
D_{x x} \simeq x^{4} y^{2} f_{x x}, \quad D_{x y} \simeq x^{3} y^{3} f_{x y}, \quad D_{y y} \simeq x^{2} y^{4} f_{y y} \quad(x, y \rightarrow 0) .
$$

where now $f_{i j}$ are simply constants. Substituting these into (5.3) and seeking the appropriate scalings reveals that the scalar in the corners will have spatial scales $x, y=O\left(\kappa^{1 / 6}\right)$ and, from consideration of the time-derivative, the decay rate will be of order $\gamma=O\left(\kappa^{2 / 3}\right)$ as seen in line (iv) of the table.

Finally we observe that the decay of large-scale fields may also be brought into this framework using a Bloch wavenumber formalism. We consider scalar fields of the general form

$$
\sigma(\boldsymbol{r}, t)=e^{i M x+i N y} \sum_{m, n} \hat{\sigma}_{m, n}(t) e^{i m x+i n y}+\text { c.c. },
$$

with $0<M, N \leq 1 / 2$. For small $M, N$ and our flows in the square domain, we obtain an isotropic transport of scalar fields on large scales, with a decay rate of the form

$$
\gamma(\kappa) \simeq \kappa_{\mathrm{eff}}(\kappa)\left(M^{2}+N^{2}\right) \quad(M, N \rightarrow 0),
$$

where $\kappa_{\text {eff }}$ is an effective diffusivity. With mixing on the advective time scale within individual cells, the transport of field on large scales is completely controlled by the flux of heat across boundary layers along the cell edges, as is evident from pictures of the scalar that we do not present here. We have already observed the controlling role of such fluxes for scalar decay with Dirichlet boundary condition, which indicates that the decay rate, say $\gamma_{\mathrm{D}}(\kappa)$, for this boundary condition should be linked to $\kappa_{\text {eff }}(\kappa)$. A straightforward calculation of fluxes links the two in the relation

$$
\kappa_{\text {eff }}(\kappa)=L^{2} \gamma_{\mathrm{D}}(\kappa) / 8 \quad(\kappa \rightarrow 0) .
$$

We have checked this result for the slip flow $\psi_{\text {slip }}(3.4)$ and the no-slip flow $\psi_{\text {no-slip }}(3.13)$, and found good agreement, which we do not present here (Zaggout, 2012).

\subsection{Numerical simulations for time-periodic flows in a square domain}

We now briefly consider the question of how well the scaling results for random flows give guidance to the behaviour of passive scalars in deterministic flows with realistic boundary 
(a)

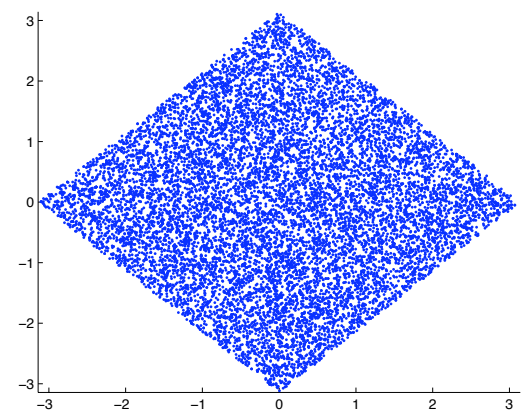

(b)

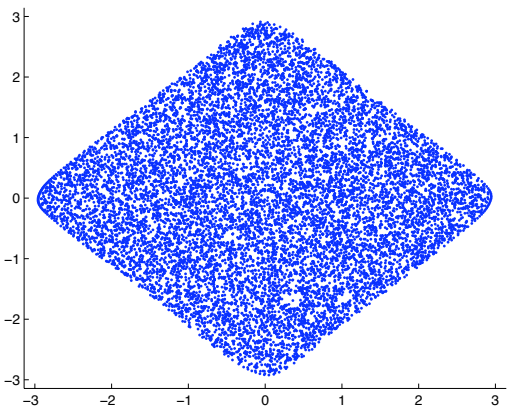

(c)

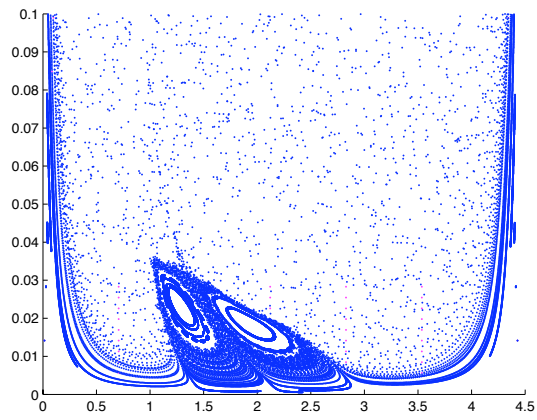

Fig. 16. Poincaré sections for the time-periodic flows (a) $\psi_{\text {slip }}$ and (b) $\psi_{\text {no-slip }}$ with $\omega=1$. Panel (c) shows a part of a section zoomed in on a segment of the boundary, using coordinates $2^{-1 / 2}(x+y+\pi)$ (horizontal) and $2^{-1 / 2}(-x+y+\pi)$ (vertical); note the small range of the vertical scale, from 0 to 0.1 , compared with the horizontal scale, from 0 to $\pi \sqrt{2}$.

conditions. Clearly we can only touch on this broad problem, and we focus on the time-periodic versions of the random flows we considered in section 3 by taking the phase $\chi(t)=0$, namely,

$$
\psi_{\text {slip }}=\phi_{1,3}(x, y) \sin \omega t+\phi_{3,1}(x, y) \cos \omega t, \quad \psi_{\text {no-slip }}=\phi_{1,3}(x, y)^{2} \sin \omega t+\phi_{3,1}(x, y)^{2} \cos \omega t
$$

with $\omega=1$. Poincaré sections for these flows are shown in figure 16. It may be seen that the phase space (within the invariant boundary $\mathcal{C}$ ) appears completely chaotic in (a) for $\psi_{\text {slip }}$. In (b) there is a thin region around the boundary $\mathcal{C}$ where the chaotic trajectories in the bulk of the domain appear not to have penetrated. In fact this region close to the boundaries is quite complicated amd panel (c) shows a Poincare section, rotated by $\pi / 4$, along the boundary joining $(-\pi, 0)$ and $(\pi, 0)$. The vertical scale is much exagerated to show structures that in fact are squashed very close to the boundary. Here it may be seen that there appear to be trajectories, with very slow motion, arbitrarily close to the boundary that then leave to enter the main chaotic region. There are also some integrable island structures. Note that our simulations below are not at a diffusivity small enough for the scalar field to 'resolve' these island structures, but the slow motion near the no-slip boundary again will be important in controlling scalar decay.

The advantage of considering periodic flow is that measuring the decay rate over one cycle is relatively easy once the field has settled into its eigenfunction of Floquet form (except when there are near-degeneracies). There is no need to take a long time average over the different possibilities in the random distribution of renewing flows. However the disadvantage is that we 
(a)

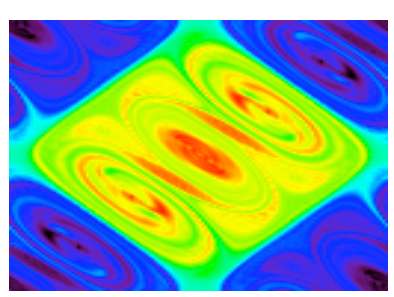

(d)

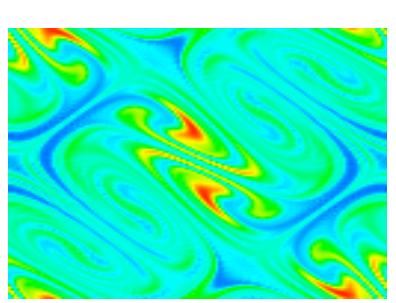

(b)

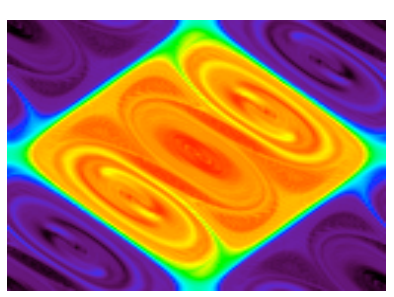

(e)

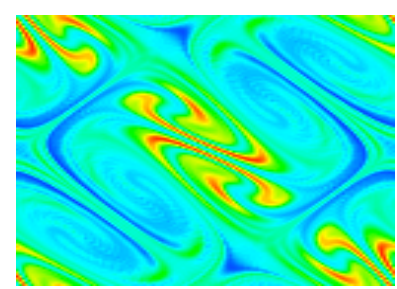

(c)

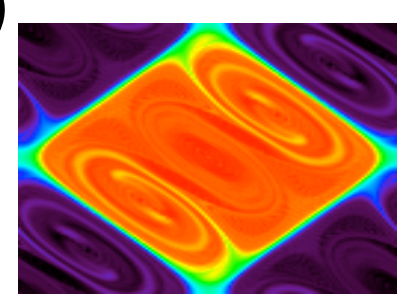

(f)

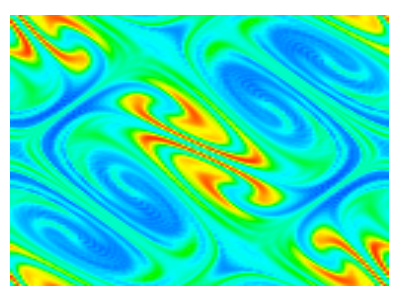

Fig. 17. Scalar field for $(\mathrm{a}-\mathrm{c})\left(\psi_{\text {no-slip }}, \sigma_{\mathrm{D}}\right)$ and $(\mathrm{d}-\mathrm{f})\left(\psi_{\text {slip }}, \sigma_{\mathrm{N}}\right)$ with $\kappa=0.001$ at $(\mathrm{a}, \mathrm{d}) t=2 \pi$, (b,e) $4 \pi$ and $(\mathrm{c}, \mathrm{f}) 6 \pi$.

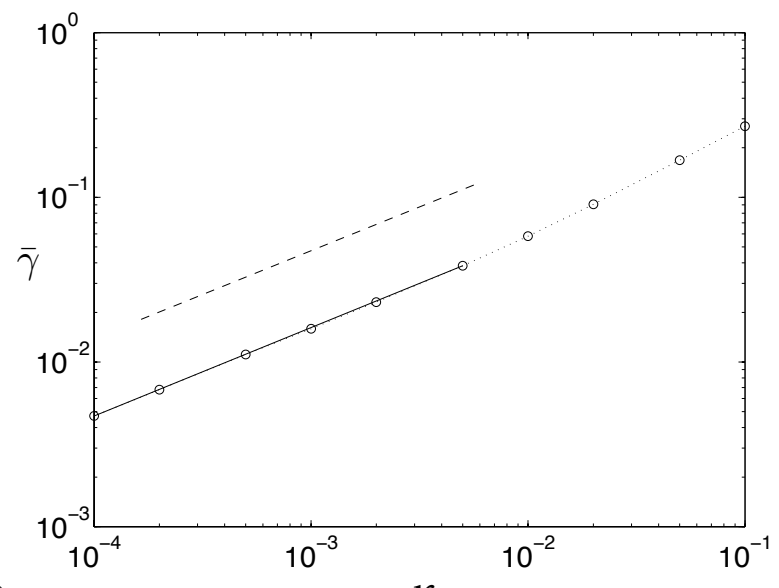

(a)

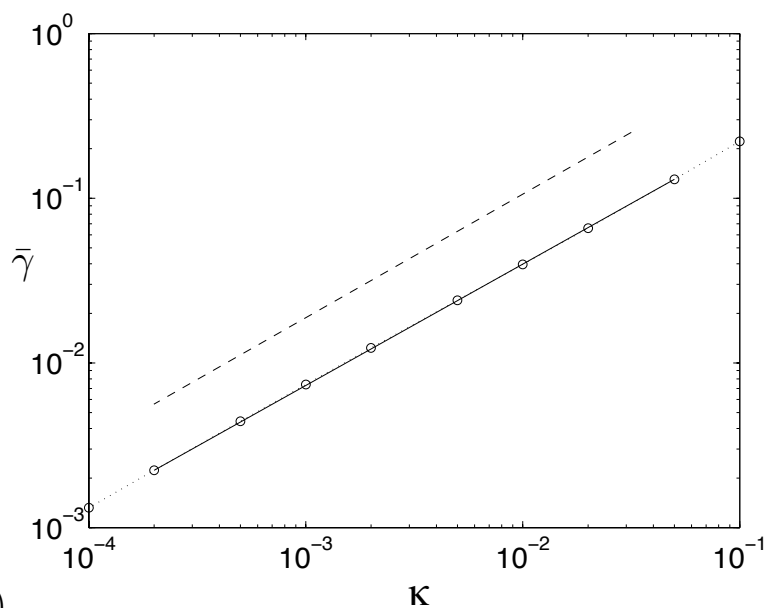

(b)
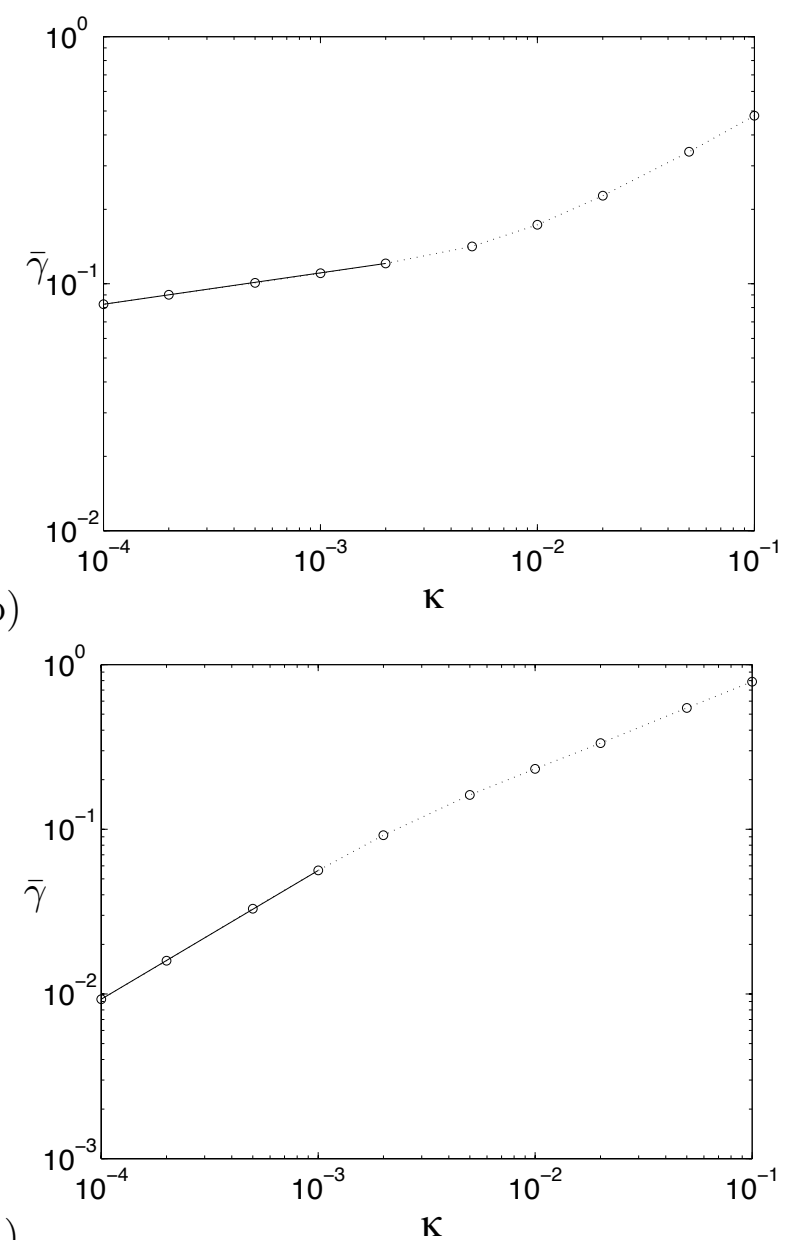

(d)

Fig. 18. Plots of $\gamma$ against $\kappa$ for the time-periodic flows. We have the cases (a) $\left(\psi_{\text {slip }}, \sigma_{\mathrm{D}}\right)$, with solid and dashed lines of slopes 0.53 and 0.5 , (b) $\left(\psi_{\text {slip }}, \sigma_{\mathrm{N}}\right)$, slope 0.126 (c) $\left(\psi_{\text {no-slip }}, \sigma_{\mathrm{D}}\right)$, slopes 0.765 and 0.75 , and $(\mathrm{d})\left(\psi_{\text {no-slip }}, \sigma_{\mathrm{N}}\right)$, slope 0.784 . 
tend to lose the clear scaling behaviour of $\gamma(\kappa)$ with $\kappa$, and we do no have theory to fall back on. For these deterministic periodic flows the symmetry properties discussed in the random case continue to hold for the initial conditions $\sigma_{\mathrm{D}}$ and $\sigma_{\mathrm{N}}$ and we need not consider this further.

Figure 17 shows sample runs of scalar field with (a-c) no-slip and Dirichlet boundary conditions, and $(\mathrm{d}-\mathrm{f})$ slip and Neumann conditions. The morphology of the field is similar to that in the random case, in figures 5 and $6(\mathrm{a}-\mathrm{c})$. We are showing stroboscopic pictures, at the first few multiples of the period $2 \pi$ of the flow and one can see the build-up of structure on small scales throughout the domain in $(\mathrm{d}-\mathrm{f})$ or the eradication of scalar fluctuations leaving only boundary layers in $(\mathrm{a}-\mathrm{c})$. Further reductions in diffusivity would lead to field structure on all scales, a so-called strange eigenfunction. Scaling laws for the four key cases are shown in figure 18 for $\psi_{\text {slip }}$ or $\psi_{\text {no-slip }}$ and Dirichlet or Neumann conditions, and we may compare these with the corresponding random flows.

The closest points of comparison are for the panels 18(a,c) which show exponents $\alpha \simeq 0.5$ and $\alpha \simeq 0.75$ for Dirichlet boundary condition with slip and no-slip flows respectively. These exponents are in good agreement with the values in table 1 for random flows. However we should note that in the case of the no-slip flow while the observed scaling is very clear, it is possible that it will change as $\kappa \rightarrow 0$ in view of the Poincaré section figure $16(\mathrm{c})$. As $\kappa$ is reduced the $\kappa^{1 / 4}$ scale of the scalar field boundary layer will become comparable with the islands near the boundary apparent in 16(c); transfer of scalar to and from the boundary may well become poorer and the value of $\alpha$ is likely to increase.

For the slip case with Neumann boundary conditions the log-log plot in figure 18(b) shows a curve flattening to a very shallow power law with exponent $\alpha \simeq 0.126$. The result for the corresponding random flow is $\alpha=0$ and at present we cannot conclude whether there really is a weak power law scaling or not present in 18(b), in particular given the small range of scale on the vertical axis. Figure 18(d) for the no-slip flow and Neumann boundary condition shows another bent curve with slope increasing from around 0.5 to $\alpha \simeq 0.75$ for small $\kappa$. This is a rather steeper power law than the value $\alpha \simeq 2 / 3$ for the random case. The decay of scalar fluctuations is controlled by a reservoir of scalar in the very sluggish flow near the corners (seen clearly in the random case in figure 6(c-f)). Plots of the scalar distribution for the deterministic case (not shown) show a very similar picture but with a greater contrast between regions of cold scalar in the corners and the well-mixed warm fluid in the bulk of the container. The transport of scalar in and out of the corners will be quite sensitive to the flows generated there (as we also pick up in the discussion below). Our results appear to indicate that the random flow is more effective at transporting scalar in and out, whereas the periodic flow is, presumably because of averaging, much poorer at persistent transport. This remains to be investigated thoroughly by the study of families of deterministic flows with varying behaviour in the corners and on the no-slip boundaries.

\section{Discussion and outlook}

We have investigated transport of passive scalars in random and time-periodic flows with slip and no-slip boundary conditions, in a plane layer and in a square, under Dirichlet and Neumann 
boundary conditions. Our results for decay rate exponents $\alpha$ for random flows are summarised in table 1 in section 5. These results confirm those of Lebedev \& Turitsyn (2004) and Salman \& Haynes (2007), and extend them to consideration of a whole variety of boundary conditions, and square as well as plane layer domains. We have found clear scaling in our numerical results, which led to conjectured values of the scaling exponent $\alpha$ backed up by theoretical discussion. Where there has been an explicit formula for decay rates with no-slip boundary conditions, we have seen good agreement between our numerical results for a single, long realisation of the random flow and the theoretical values based on an ensemble average in figure $15(\mathrm{a}, \mathrm{b})$.

Our study imposes boundary conditions on the flow and scalar field by means of symmetries which allows a unified approach, together with efficient numerical simulations based solely in spectral space. We have not pushed $\kappa$ to extremely low values, preferring to explore a wide range of values and use long runs, but this could be done effectively with the flow fields we have defined. As we discussed, this use of symmetries means we can apply exact Dirichlet and Neumann boundary conditions for the flow fields with slip along the boundary. In the case of noslip flows, these boundary conditions are only imposed approximately as we lose the reflection symmetries about the walls, to be replaced by rotation symmetries. With temperature as the scalar field under consideration, we note that the boundary conditions used correspond to a thin walls between neighbouring cells of fluid flow with appropriate temperature fields and so are physically sensible. Furthermore when theory is developed by ensemble averaging over the random velocity fields, the resulting effective diffusion tensor $D_{i j}$ regains these reflectional symmetries (given that the averaged fields have these symmetries in a statistical sense), and so we regain exact Dirichlet and Neumann boundary conditions for the no-slip case. Further investigations of the equations (5.3) with the full diffusion tensor $D_{i j}(x, y)$ in a square domain bear this out (Zaggout, 2012). In short, while our results in the no-slip case do not use exact Dirichlet and Neumann boundary conditions, we would argue that the power-law exponents $\alpha$ will be the same in the exact case. Physically, the very slow time scales in the boundary layer lead to an effective average over the rapid motion in the bulk and recover the exact boundary conditions as a leading order approximation.

One of our interests in this work was to understand the role of corner regions in a square domain, by comparison with the infinite plane layers considered by Lebedev \& Turitsyn (2004) and Salman \& Haynes (2007). We find that the corners have a largely passive role except in the case of the Neumann boundary condition for a no-slip flow. Here the corner regions act as reservoirs, where slow motion leads to slow release of scalar, controlling the decay of fluctuations in the bulk for small diffusivity $\kappa$ : the mechanism is the same as that identified for the plane layer boundaries (Lebedev \& Turitsyn, 2004), but the time-scale is still slower, leading to an $\alpha=2 / 3$ exponent rather than $\alpha=1 / 2$, for the flows we have used.

We should note however that in applications the flows in corner regions (in two and three dimensions) can be rather sensitive to how a fluid flow is driven, something we have not considered in this paper. For example, if a very viscous fluid motion is driven in a two-dimensional box by means of a periodically oscillating lid, near the corners the flow possesses an infinite sequence of Moffatt or corner eddies (Moffatt, 1964; Shankar, 2007). In this case locally the flow takes the form

$$
\psi(r, \theta)=C r^{\lambda} f(\theta)+\text { c.c. },
$$


in polar coordinates $(r, \theta)$ based at the corner, and for a corner of semi-angle $\beta$, the exponent $\lambda$ satisfies

$$
(\lambda-1) \sin 2 \beta+\sin 2(\lambda-1) \beta=0 .
$$

For example for a right-angled corner as we have, $\beta=\pi / 4$ and $\lambda \simeq 3.73959+1.11902 i$, this corresponding to more motion (slower fall-off in radius) than we have in our models as the corner is approached; see (5.15), equivalent to $\lambda=4$.

For a random flow, suppose we now average over random corner eddy configurations, and suppose that the phases of the constant $C$ in (6.1) are uniformly distributed between 0 and $2 \pi$. We will obtain a diffusivity tensor $D_{i j} \propto r^{2 \lambda_{\mathrm{r}}-2}$ with $\lambda_{\mathrm{r}}$ the real part of $\lambda$. This gives a corner boundary layer of scale $x, y=O\left(\kappa^{1 /\left(2 \lambda_{\mathrm{r}}-2\right)}\right)$ and, from consideration of the timederivative, the decay rate will be $\gamma=O\left(\kappa^{\alpha}\right)$ with $\alpha=\left(\lambda_{\mathrm{r}}-2\right) /\left(\lambda_{\mathrm{r}}-1\right)$. Our previous results in (5.11) correspond to $\lambda_{\mathrm{r}}=4$ and $\alpha=2 / 3$, but for Moffatt eddies in a right-angled corner we have $\lambda_{\mathrm{r}} \simeq 3.73959$, giving faster decay, $\alpha \simeq 0.63498$. If the semi-angle $\beta$ of the corner is increased we obtain yet more rapid decay of passive scalars; for example moving to a hexagonal shaped container, the angle $\beta$ is $\pi / 3$ and $\lambda_{\mathrm{r}} \simeq 3.09414$, giving further decrease in the scaling exponent to $\alpha \simeq 0.52248$. If on the other hand the semi-angle is reduced decay is suppressed; for instance for a triangular container, $\beta=\pi / 6, \lambda_{\mathrm{r}} \simeq 5.05933$ and the decay is much reduced, with $\alpha \simeq 0.75365$. Note that if the values of $C$ in (6.1) are random but the phases are not uniformly distributed, then a memory of the radial eddy structure may remain in $D_{i j}$ which will contain a power law dependence on $r$ superposed with oscillations in $\log r$. We may then expect the decay rate itself to show a dependence with power law decay and oscillations as $\kappa$ is reduced. This theoretical direction remains to be explored in the context of suitable flows, for example in a cavity driven by a lid with appropriate random motions.

\section{Acknowledgements}

We are grateful to Jean-Luc Thiffeault, John Thuburn and Matthew Turner for valuable discussions and references, and to the anonymous referees for their useful comments and suggestions. FZ acknowledges support from the Libyan Government in the form of a research studentship.

\section{References}

Antonsen, T.M., Fan, Z., Ott, E. \& Garcia-Lopez, E. 1996 The role of chaotic orbits in the determination of power spectra of passive scalars. Phys. Fluids 8, 3094-3104.

Aref, H. 1984 Stirring by chaotic advection. J. Fluid Mech. 143, 1-21.

Balmforth, N.J. \& Young, W.R. 2003 Diffusion-limited scalar cascades. J. Fluid Mech. 482, 91-100.

Boffetta, G., De Lillo, F. \& Mazzino, A. 2009 Peripheral mixing of passive scalar at small Reynolds number. J. Fluid Mech. 624, 151-158.

Burghelea, T., Segre, E., Bar--Joseph, I., Groisman, A. \& Steinberg, V. 2004 Chaotic flow and efficient mixing in a microchannel with a polymer solution. Phys. Rev. E 69, 066305. 
Burghelea, T., Segre, E. \& Steinberg, V. 2004 Mixing by polymers: experimental test of decay regime of mixing. Phys. Rev. Lett. 92, 164501.

Camassa, R., Martinsen-Burrell, N. \& McLaughlin, R.M. 2007 Dynamics of probability density functions for decaying passive scalars in periodic velocity fields. Phys. Fluids 19, 117104.

Cerbelli, S., Adrover, A. \& Giona, M. 2003 Enhanced diffusion regimes in bounded chaotic flows. Phys. Lett. A 312, 355-362.

Chernykh, A. \& Lebedev, V. 2008 Passive scalar structures in peripheral regions of random flows JETP Letters 87, 682-685.

Chertkov, M. \& Lebedev, V. 2003 Decay of scalar turbulence revisited. Phys. Rev. Lett. 90, 034501.

El Omari, K. \& Le Guer, Y. 2010 Alternate rotating walls for thermal chaotic mixing. Int. J. Heat Mass Transfer 53, 123-134.

Fereday, D.R. \& Haynes, P.H. 2004 Scalar decay in two-dimensional chaotic advection and Batchelor-regime turbulence. Phys. Fluids 16, 4359-4370.

Garcia-Ybarra, P.L. 2009 Near-wall turbulent transport of large-Schmidt-number passive scalars. Phys. Rev. E 79, 067302.

Gilbert, A.D. 2006 Advected fields in maps III: passive scalar decay in baker's maps. Dyn. Sys. 21, 25-71.

Giona, M., Adrover, A., Cerbelli, S. \& Vitacolonna, V. 2004 Spectral properties and transport mechanisms of partially chaotic bounded flows in the presence of diffusion. Phys. Rev. Lett. 2004, 11401.

Gouillart, E., Dauchot, O., Dubrulle, B., Roux, S. \& Thiffeault, J.-L. 2008 Slow decay of concentration variance due to no-slip walls in chaotic mixing. Phys. Rev. E 78, 026211.

Gouillart, E., Kuncio, N. Dauchot, O., Dubrulle, B., Roux, S. \& Thiffeault, J.-L. 2007 Walls inhibit chaotic mixing. Phys. Rev. Lett. 99, 114501.

Gouillart, E., Thiffeault, J.-L. \& Dauchot, O. 2010 Rotation shields chaotic mixing regions from no-slip walls. Phys. Rev. Lett. 104, 204502.

Groisman, A. \& Steinberg, V. 2001 Efficient mixing at low Reynolds numbers using polymer additives. Nature 410, 905-908.

Haynes, P.H.\& Vanneste. J. 2005 What controls the decay of passive scalars in smooth flows? Phys. Fluids 17, 097103.

Jun, Y. \& Steinberg, V. 2010 Mixing of passive tracers in the decay Batchelor regime of a channel flow. Phys. Fluids 22, 123101.

Lebedev, V.V. \& Turitsyn, K.S. 2004 Passive scalar evolution in peripheral regions. Phys. Rev. E 69, 036301.

Moffatt, H.K. 1964 Viscous and resistive eddies near a sharp corner. J. Fluid Mech. 18, 1-18.

Ottino, J.M. 1989 The kinematics of mixing: stretching, chaos, and transport. Cambridge University Press.

Pierrehumbert, R.T. 1994 Tracer microstructure in the large-eddy dominated regime. Chaos, Solitons \& Fractals 4, 1091-1110.

Pierrehumbert, R.T. \& Yang, H. 1993 Global chaotic mixing on isentropic surfaces J. Atmos. Sci. $502462-2462$.

Plunian, F., Marty, P. \& Alemany, A. 1999 Kinematic dynamo action in a network of screw motions; application to the core of a fast breeder reactor J. Fluid Mech. 382, 137-154.

Salman, H. \& Haynes, P. H. 2007 A numerical study of passive scalar evolution in peripheral 
regions. Phys. Fluids 19, 067101-1.

Schekochihin, A.A., Haynes, P.H. \& Cowley, S.C. 2004 Diffusion of passive scalar in a finitescale random flow. Phys. Rev. E 70, 046304.

Shankar, P.N. 2007 Slow viscous flows. Imperial College Press.

Simmonet, C. \& Groisman, A. 2005 Chaotic mixing in a steady flow in a microchannel. Phys. Rev. Lett. 94, 134501.

Skvortsov, A., \& Yee, E. 2011 Scaling laws of peripheral mixing of passive scalar in a wall-shear layer. Phys. Rev. E 83, 036303.

Toussaint, V., Carriere, P., Scott, J. \& Gence, J.-N. 2000 Spectral decay of a passive scalar in chaotic mixing. Phys. Fluids 12, 50011.

Turner, M.R., Thuburn, J. \& Gilbert, A.D. 2009 The influence of periodic islands in the flow on a scalar tracer in the presence of a steady source. Phys. Fluids 21, 067103.

Zaggout, F. 2012 Passive scalar mixing in chaotic flows with boundaries. PhD Thesis, University of Exeter. 